\title{
Ground state properties and bubble structure of synthesized superheavy nuclei
}

\author{
S. K. Singh * \\ Institute of Physics, Bhubaneswar-751 005, India. \\ M. $\operatorname{Ikram}^{\dagger}$ \\ Department of Physics, Aligarh Muslim University, Aligarh-202002, India. \\ S. K. Patra $\ddagger$ \\ Institute of Physics, Bhubaneswar-751 005, India.
}

\begin{abstract}
We calculate the ground state properties of recently synthesized superheavy elements from $Z=105-118$ along with the predicted proton magic $Z=120$. The relativistic and nonrelativistic mean field formalisms are used to evaluate the binding energy, charge radius, quadrupole deformation parameter and the density distribution of nucleons. We analyzed the stability of the nuclei based on the binding energy and neutron to proton ratio. We also studied the bubble structure which reveals the special features of the superheavy nuclei.
\end{abstract}

\section{Introduction}

The study of superheavy elements (SHEs) is an interesting topic for the current days research in nuclear physics. Initially, the transuranium elements were made by subjecting Uranium to a high neutron flux allowing to capture neutrons successively. The neutron-rich isotopes undergo $\beta$-decay and produce new elements. This process allows to prepare element maximum upto Fermion $(Z=100)$. This is due to the shorter time interval of the spontaneous fission than neutron capture time of the newly form element. To make heavier $Z$ nuclei one has to reach the so called island of stability which is a matter of discussion from last five decades 1/2/3/4. Earlier, it was predicted 115 that the next proton magic number beyond $\mathrm{Z}=82$ would be 126 considering the equality of the proton and neutron magic numbers for known closed shell nuclei. However, several microscopic calculations $6[7 / 8 / 9|10| 11$ suggest a shift of this number to 114. One of the cause of the shift is the Coulomb effect on the spherical single particle levels. The use of shell correction by V. M. Strutinsky 12 to the liquid-drop calculation of binding energy (BE) opens a more satisfactory exploration towards the search of double close nucleus beyond ${ }^{208} \mathrm{~Pb}$. Using this

*S. K. Singh, Email: shailesh@iopb.res.in

${ }^{\dagger}$ M. Ikram, Email: ikram@iopb.res.in

${ }^{\ddagger}$ Dr. S. K. Patra, Email: patra@iopb.res.in 
approach, $\mathrm{Z}=114$ is supported to be the proton magic after 82 in Refs. $13[14 \mid 15] 16$. Based on the relativistic mean field (RMF) and Skyrme Hartree-Fock (SHF) calculations $17|18| 19|20| 21$, it is predicted that the island of stability in the superheavy region is situated near mass number $A \sim 300$ peaking at ${ }^{292,304} 120$.

The main aim is to reach the stability island of superheavy valley using some alternative process rather than the nucleosynthesis by neutron capture. Using cold fusion reaction, elements from $Z=107-112$ are synthesized at GSI $22|23| 24|25| 26|27| 28$ which are based on $\mathrm{Pb}$ and $\mathrm{Bi}$ targets. At the production time of $\mathrm{Z}=112$ nucleus at GSI, the fusion cross section was extremely small $(1 \mathrm{pb}) 26$, which led to the conclusion that reaching still heavier elements will be very difficult by this process. The element $\mathrm{Z}=113$ was also synthesized in coldfusion reaction at RIKEN with a very low cross section $\sim 0.03 \mathrm{pb} 29$ confirming the limitation of cold-fusion synthesis.

To overcome this problem in hot fusion evaporation reactions with a deformed actinide targets and a neutron-rich doubly magic spherical projectile like ${ }^{48} \mathrm{Ca}$ are used in the synthesis of superheavy nuclei $Z=112-118$ at Dubna 30|31|32|33|34|35. It is worthwhile to mention that, although the synthesis of SHEs is a challenging task, the knowledge of their chemistry for the recently synthesized nuclei $(\mathrm{Z}=104$ 118 ) are more interesting to find the analogies between the properties of new elements and their lighter known neighbours in the groups of chemical elements and, eventually, proving their position in the periodic table. Nowadays, the chemistry of some trans-actinide elements, like $\mathrm{Z}=107,108,112$ and 114 are known experimentally $36 / 37 / 38 / 39 \mid 40$ and theoretical efforts are also given 41 . These elements are located at the bottom of the periodic table. These nuclei are extremely unstable and the electron shells are influenced by strong relativistic effects 42|43|44|45|46].

The stability of a nucleus mostly depends on its structure which is again a function of the combination of neutrons and protons number. Because of the large Coulombic repulsion, the central portion of the nucleus may take a depletion and create a bubble like structure. There are some interesting and extensive calculations for various properties including bubbles or semi-bubbles structure of superheavy elements 47 48. However, theoretical predictions are in active discussion stage for the ground state structure of these double close nuclei $\mathrm{Z}=114,120$ or 126 and $\mathrm{N}=172$ or 184 . For example, Z. Ren et al. $\frac{49 \mid 50}{4}$ predicted the nuclei have a well deformed ground state in RMF approach. Of course a spherical degenerate state is also available in this calculations. In contrast to the deform configuration of Ref. 4950, Muntian et al. $\frac{51}{1}$ find a spherical shape in the macro-microscopic approach. In the frame-work of SHF with SkP or SLy7 interaction Cwiok et al ${ }^{52}$ find ${ }^{310} 126$ as a spherical double close shell nucleus. In this paper, the stability and bubble structure of the superheavy nuclei are discussed by two well known self-consistent mean field models, namely Skyrme Hartree-Fock (SHF) using SkI4 parameters 53 with BCS pairing and Relativistic Mean Field (RMF) model with NL3* parameter set $\underline{54}$. 
The paper starts with a short introduction in Sec. I. The formalisms of SHF and RMF are presented in Sec.II. Mostly, the stability and structure of the synthesized nuclei will be discussed in Sec. III. The bubble properties will also be analyzed taking into account the density distribution of protons and neutrons. Finally a brief summary and concluding remarks will be given in Sec. IV.

\section{Theoretical Formalisms}

\subsection{Skyrme Hartree-Fock (SHF) method}

There are many known parametrization of Skyrme interaction which reproduce the experimental data for ground state properties of finite nuclei $[55156$ as well as the properties of infinite nuclear matter upto high density 57 . The general form of the Skyrme effective interaction can be expressed as a density functional $\mathcal{H}$ with some empirical parameters $55158 \mid 59$.

$$
\mathcal{H}=\mathcal{K}+\mathcal{H}_{0}+\mathcal{H}_{3}+\mathcal{H}_{\text {eff }}+\cdots,
$$

where $\mathcal{K}$ is the kinetic energy, $\mathcal{H}_{0}$ the zero range, $\mathcal{H}_{3}$ the density dependent and $\mathcal{H}_{\text {eff }}$ the effective-mass dependent terms, which are relevant for calculating the properties of nuclear matter. More details can be found in Refs. 55/58|59. These are functions of 9 parameters $t_{i}, x_{i}(i=0,1,2,3)$ and $\eta$ are given as

$$
\begin{aligned}
\mathcal{H}_{0}= & \frac{1}{4} t_{0}\left[\left(2+x_{0}\right) \rho^{2}-\left(2 x_{0}+1\right)\left(\rho_{p}^{2}+\rho_{n}^{2}\right)\right], \\
\mathcal{H}_{3}= & \frac{1}{24} t_{3} \rho^{\eta}\left[\left(2+x_{3}\right) \rho^{2}-\left(2 x_{3}+1\right)\left(\rho_{p}^{2}+\rho_{n}^{2}\right)\right], \\
\mathcal{H}_{\text {eff }}= & \frac{1}{8}\left[t_{1}\left(2+x_{1}\right)+t_{2}\left(2+x_{2}\right)\right] \tau \rho \\
& +\frac{1}{8}\left[t_{2}\left(2 x_{2}+1\right)-t_{1}\left(2 x_{1}+1\right)\right]\left(\tau_{p} \rho_{p}+\tau_{n} \rho_{n}\right) .
\end{aligned}
$$

The kinetic energy $\mathcal{K}=\frac{\hbar^{2}}{2 m} \tau$, a form used in the Fermi gas model for non-interacting Fermions. The other terms $\mathcal{H}_{\mathcal{S} \rho}$ and $\mathcal{H}_{\mathcal{S} \overrightarrow{\mathcal{J}}}$ with $b_{4}$ and $b_{4}^{\prime}$ are the surface contributions which also responsible for a better spin-orbit interaction are defined as

$$
\begin{aligned}
\mathcal{H}_{S \rho}= & \frac{1}{16}\left[3 t_{1}\left(1+\frac{1}{2} x_{1}\right)-t_{2}\left(1+\frac{1}{2} x_{2}\right)\right](\vec{\nabla} \rho)^{2} \\
& -\frac{1}{16}\left[3 t_{1}\left(x_{1}+\frac{1}{2}\right)+t_{2}\left(x_{2}+\frac{1}{2}\right)\right] \\
& \times\left[\left(\vec{\nabla} \rho_{n}\right)^{2}+\left(\vec{\nabla} \rho_{p}\right)^{2}\right], \text { and } \\
\mathcal{H}_{S \vec{J}}= & -\frac{1}{2}\left[b_{4} \rho \vec{\nabla} \cdot \vec{J}+b_{4}^{\prime}\left(\rho_{n} \vec{\nabla} \cdot \overrightarrow{J_{n}}+\rho_{p} \vec{\nabla} \cdot \overrightarrow{J_{p}}\right)\right] .
\end{aligned}
$$

Here, the total nucleon number density $\rho=\rho_{n}+\rho_{p}$, the kinetic energy density $\tau=\tau_{n}+\tau_{p}$, and the spin-orbit density $\vec{J}=\vec{J}_{n}+\vec{J}_{p}$. The subscripts $n$ and $p$ refer to 
neutron and proton, respectively, and $M$ is the nucleon mass. The $\vec{J}_{q}=0, q=n$ or $p$, for spin-saturated nuclei, i.e., for nuclei with major oscillator shells completely filled. The total binding energy (BE) of a nucleus is the integral of the density functional $\mathcal{H}$. In our calculations, we have used the Skyrme SkI4 parameter set 53 . This set is known to well account the spin-orbit interaction in finite nuclei, related to the isotope shifts in $\mathrm{Pb}$ region and is better suited for the study of exotic nuclei. Several more recent Skyrme parameters such as SLy1-10, SkX, SkI5 and SkI6 are obtained by fitting the Hartree-Fock (HF) results with experimental data for nuclei starting from the valley of stability to neutron and proton drip-lines 53158160161 .

\subsection{Relativistic mean field (RMF) formalism}

From last few decades, the RMF theory is applied successfully to study the structural properties of nuclei throughout the periodic table $\frac{62|63| 64|65| 66}{6}$ starting from proton to neutron drip-lines. The starting point of the RMF theory is the basic Lagrangian containing nucleons interacting with $\sigma-, \omega-$ and $\rho-$ meson fields. The photon field $A_{\mu}$ is included to take care of the Coulomb interaction of protons. The relativistic mean field Lagrangian density is expressed as 62[63[64|65|66,

$$
\begin{aligned}
\mathcal{L} & =\bar{\psi}_{i}\left\{i \gamma^{\mu} \partial_{\mu}-M\right\} \psi_{i}+\frac{1}{2} \partial^{\mu} \sigma \partial_{\mu} \sigma-\frac{1}{2} m_{\sigma}^{2} \sigma^{2}-\frac{1}{3} g_{2} \sigma^{3} \\
& -\frac{1}{4} g_{3} \sigma^{4}-g_{s} \bar{\psi}_{i} \psi_{i} \sigma-\frac{1}{4} \Omega^{\mu \nu} \Omega_{\mu \nu}+\frac{1}{2} m_{w}^{2} V^{\mu} V_{\mu} \\
& -g_{w} \bar{\psi}_{i} \gamma^{\mu} \psi_{i} V_{\mu}-\frac{1}{4} \vec{B}^{\mu \nu} \vec{B}_{\mu \nu}+\frac{1}{2} m_{\rho}^{2} \vec{R}^{\mu} \vec{R}_{\mu}-\frac{1}{4} F^{\mu \nu} F_{\mu \nu} \\
& -g_{\rho} \bar{\psi}_{i} \gamma^{\mu} \vec{\tau} \psi_{i} \overrightarrow{R^{\mu}}-e \bar{\psi}_{i} \gamma^{\mu} \frac{\left(1-\tau_{3 i}\right)}{2} \psi_{i} A_{\mu} .
\end{aligned}
$$

Here $\mathrm{M}, m_{\sigma}, m_{\omega}$ and $m_{\rho}$ are the masses for nucleon, $\sigma$-, $\omega$ - and $\rho$-mesons and $\psi$ is its Dirac spinor. The field for the $\sigma$-meson is denoted by $\sigma, \omega$-meson by $V_{\mu}$ and $\rho$-meson by $R_{\mu} . g_{s}, g_{\omega}, g_{\rho}$ and $e^{2} / 4 \pi=1 / 137$ are the coupling constants for the $\sigma$, $\omega, \rho$-mesons and photon respectively. $g_{2}$ and $g_{3}$ are the self-interaction coupling constants for $\sigma$ mesons. By using the classical variational principle we obtain the field equations for the nucleons and mesons.

$$
\begin{aligned}
\left\{-\triangle+m_{\sigma}^{2}\right\} \sigma^{0}\left(r_{\perp}, z\right) & =-g_{\sigma} \rho_{s}\left(r_{\perp}, z\right) \\
& -g_{2} \sigma^{2}\left(r_{\perp}, z\right)-g_{3} \sigma^{3}\left(r_{\perp}, z\right) \\
\left\{-\triangle+m_{\omega}^{2}\right\} V^{0}\left(r_{\perp}, z\right) & =g_{\omega} \rho_{v}\left(r_{\perp}, z\right) \\
\left\{-\triangle+m_{\rho}^{2}\right\} R^{0}\left(r_{\perp}, z\right) & =g_{\rho} \rho_{3}\left(r_{\perp}, z\right), \\
-\triangle A^{0}\left(r_{\perp}, z\right) & =e \rho_{c}\left(r_{\perp}, z\right) .
\end{aligned}
$$

The Dirac equation for the nucleons is written by

$$
\left\{-i \alpha . \nabla+V\left(r_{\perp}, z\right)+\beta M^{\dagger}\right\} \psi_{i}=\epsilon_{i} \psi_{i} .
$$


The effective mass of the nucleon is

$$
M^{\dagger}=M+S\left(r_{\perp}, z\right)=M+g_{\sigma} \sigma\left(r_{\perp}, z\right),
$$

and the vector potential is

$$
V\left(r_{\perp}, z\right)=g_{\omega} V^{0}\left(r_{\perp}, z\right)+g_{\rho} \tau_{3} R^{0}\left(r_{\perp}, z\right)+e \frac{\left(1-\tau_{3}\right)}{2} A^{0}\left(r_{\perp}, z\right) .
$$

A static solution is obtained from the equations of motion to describe the ground state properties of nuclei. The set of nonlinear coupled equations are solved selfconsistently in an axially deformed harmonic oscillator basis $N_{F}=N_{B}=20$. The quadrupole deformation parameter $\beta_{2}$ is extracted from the calculated quadrupole moments of neutrons and protons through

$$
Q=Q_{n}+Q_{p}=\sqrt{\frac{16 \pi}{5}}\left(\frac{3}{4 \pi} A R^{2} \beta_{2}\right)
$$

where $\mathrm{R}=1.2 A^{1 / 3}$.

The total energy of the system is given by

$$
E_{\text {total }}=E_{\text {part }}+E_{\sigma}+E_{\omega}+E_{\rho}+E_{c}+E_{\text {pair }}+E_{c . m .},
$$

where $E_{\text {part }}$ is the sum of the single particle energies of the nucleons and $E_{\sigma}, E_{\omega}, E_{\rho}$, $E_{c}, E_{\text {pair }}, E_{c m}$ are the contributions of the meson fields, the Coulomb field, pairing energy and the center-of-mass energy, respectively. We use the recently reported NL3* parameter set $\stackrel{54}{ }$ in our calculations for RMF formalism.

\section{Results and discussions}

In the present paper, we calculate the binding energy, root mean square (rms) charge radius $r_{c h}$ and quadrupole deformation parameter $\beta_{2}$. A special attention is given to the neutron and proton density distributions of these superheavy nuclei, where we search for the bubble structure at various excited intrinsic states. The results are explained in subsection 3.1 to 3.5.

\subsection{Stability of nuclei}

While analyzing the nuclear landscape, the stability of nucleus is maximum with a neutron to proton ratio $N / Z$ at about 1 for light mass region. This value of $N / Z$ increases with mass number A to neutralize the Coulomb repulsion and saturated at $\sim 1.5$ for naturally occuring nuclei. For example, the neutron to proton ratio for ${ }^{208} \mathrm{~Pb}$ is 1.537 and for ${ }^{235,238} \mathrm{U}$, these values are 1.554 and 1.587 , respectively. To see the overall $\mathrm{N} / \mathrm{Z}$ values of the synthesized isotopes in the superheavy region, we calculate the neutron to proton ratio for $Z=105$ to 118 in addition to the predicted double close nuclei ${ }^{292,293,304} 120$. We find the minimum and maximum $N: Z$ as 1.433 and 1.614 , respectively.

One important quantity is the binding energy $(B E)$ of a nucleus. The stability of a nucleus does not fully depend on the binding energy only. The arrangement 
of shells inside the nucleus is also a function of stability. In the absence of shell energy contribution (liqiud-drop limit), it may be possible that a general view on stability of nuclei can be made on the basis of binding energy per particle $(B E / A)$, i.e., nucleus is more stable with greater value of $B E / A$. Binding energy is one of the experimental observable, which can be measured precisely and could be an important input for stability. Generally, a nucleus is more stable with a larger binding energy per particle along an isotopic chain. The trend of $B E / A$ as a function of mass number A is shown in Figure 1 It is clear that low mass nuclei in the vicinity of $\beta$-stability valley have more life-time compare to the heavier isotopes. This may be due to the large Coulomb repulsion of the superheavy nuclei, although the $N / Z$ ratio is almost constant. The declining trend of $B E / A$ indicates the difficulty in the formation of heavier superheavy nuclei. As we have mentioned, not only the BE, but also the internal shell structure plays for the role of stability. Thus, there is a bright chance for the formation of superheavy nucleus if a proper combination of $\mathrm{N}$ and $\mathrm{Z}$ are selected which could be the next double close nucleus. In this context, it is to be recalled that the formation of a neutron-proton bound state system requires only $2.224 \mathrm{MeV}$ which is the typical binding energy of Deuteron $(B E / A \sim 1.112$ $\mathrm{MeV}) 67$.

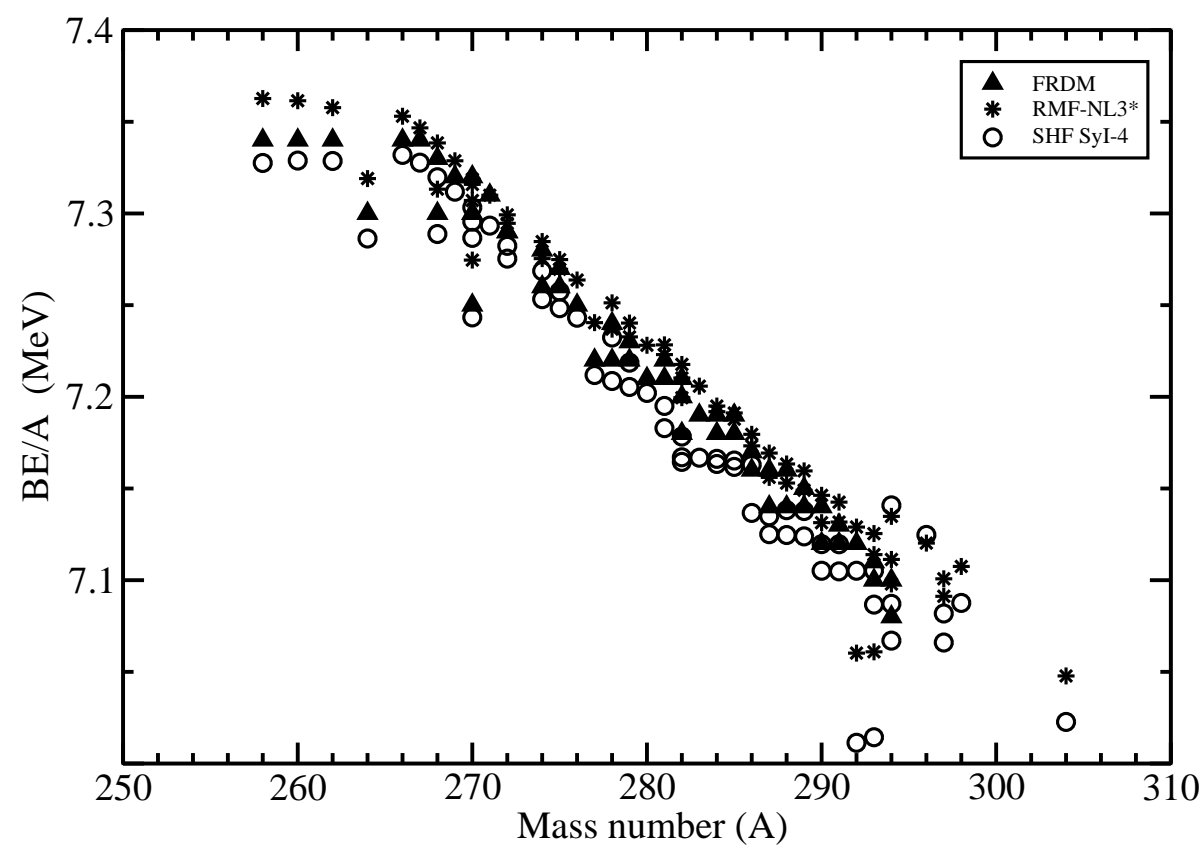

Fig. 1. The binding energy per nucleon $(B E / A)$ is compared with the FRDM data. The triangles, stars and circles are for FRDM, RMF(NL3*) and $\mathrm{SHF}(\mathrm{SkI} 4)$, respectively. 


\subsection{Binding energy and quadrupole deformation parameter}

The ground state binding energy and quadrupole deformation parameter for all the observed nuclei considered here are given in Tables I and II. The much discussed superheavy isotopes ${ }^{292,304} 120$ which are considered to be the next double closed nuclei beyond ${ }^{208} \mathrm{~Pb}$ are also listed. To find the ground state solution, the calculations are done with an initial spherical, prolate and oblate quadrupole deformation parameter $\beta_{0}$ both in the Skyrme Hartree-Fock (SHF) and relativistic mean field (RMF) formalisms. The well known SkI4 $4^{53}$ and NL3 ${ }^{254}$ parametrizations are used through out the SHF and RMF calculations, respectively. It is to be noted that the maximum binding energy corresponds to the ground state and all other solutions are the intrinsic excited state configurations. Considering this criteria, we evaluate the ground state binding energy and also the first and second excited states wherever available. The results obtained from SHF are listed in Table I and those for RMF are listed in Table II. Since the experimental binding energy of these nuclei are not known, to get a first hand information about the predictive power of our calculations, we have inserted the most commonly used binding energy of finite range droplet model (FRDM) 68. The quadrupole deformation parameter $\beta_{2}$ obtained in FRDM is also given for comparison 69. Comparing Tables I and II, it is remarkable to note that the binding energy obtained by $\mathrm{SHF}(\mathrm{SkI} 4)$ and $\mathrm{RMF}\left(\mathrm{NL} 3{ }^{*}\right)$ are quite agreeable with FRDM prediction. The deviation of our results (from the FRDM prediction) is a maximum of about $0.5 \%$. A further inspection of the results finds an edge of RMF(NL3*) over SHF(SkI4). In general, the calculated binding energies and deformation parameters are in similar trend with each other in all the three models. 
Table 1. The BE (MeV), $\beta_{2}, r_{c h}(\mathrm{fm})$ (ground state) and D.F. (\%) using SHF(SkI4).

\begin{tabular}{|c|c|c|c|c|c|c|c|c|c|c|c|c|c|c|c|}
\hline Nuclei & \multicolumn{3}{|c|}{$\overline{\mathrm{BE}}$} & \multicolumn{3}{|c|}{$\overline{\beta_{2}}$} & $r_{c h}$. & \multicolumn{2}{|c|}{ sph. } & \multicolumn{2}{|c|}{ prol. } & \multicolumn{2}{|c|}{ obl. } & \multicolumn{2}{|c|}{ FRDM } \\
\hline & sph. & prol. & obl. & sph. & prol. & obl. & & $(\mathrm{DF})_{n}$ & $(\mathrm{DF})_{p}$ & $(\mathrm{DF})_{n}$ & $(\mathrm{DF})_{p}$ & $(\mathrm{DF})_{n}$ & $(\mathrm{DF})_{p}$ & $\mathrm{BE}$ & $\overline{\beta_{2}}$ \\
\hline${ }^{266} \mathrm{Db}$ & & \begin{tabular}{|l|}
1950.3 \\
\end{tabular} & 1939.7 & & 0.262 & \begin{tabular}{|l|}
-0.282 \\
\end{tabular} & 6.08 & 0.0 & 0.0 & 0.0 & 2.98 & 1.32 & 7.86 & 953.42 & 0.221 \\
\hline${ }^{267} \mathrm{Db}$ & & 1956.5 & 1945.9 & & 0.258 & -0.283 & 6.09 & 0.0 & 3.41 & 0.0 & 3.41 & 1.29 & 7.43 & 960.49 & 0.230 \\
\hline${ }^{268} \mathrm{Db}$ & & 1961.7 & 1951.3 & & 0.254 & -0.193 & 6.09 & 0.0 & 3.42 & 0.0 & 3.83 & 1.35 & 7.36 & 965.86 & 0.221 \\
\hline${ }^{269} \mathrm{Db}$ & & 1966.9 & 1957.6 & & 0.250 & -0.191 & 6.10 & 0.0 & 3.58 & 0.0 & 3.60 & 1.13 & 7.53 & 972.01 & 0.221 \\
\hline${ }^{270} \mathrm{Db}$ & & 1971.9 & 1963.7 & & 0.259 & -0.188 & 6.11 & 0.0 & 3.65 & 0.0 & 2.12 & 0.81 & 7.64 & 976.81 & 0.212 \\
\hline${ }^{258} \mathrm{Sg}$ & & 90.5 & 1880.0 & & 0.287 & -0.287 & 6.05 & 2.73 & 4.52 & 2.73 & 4.54 & 2.74 & 0.89 & 5.22 & 0.248 \\
\hline${ }^{260} \mathrm{Sg}$ & & 1905.5 & 1894.9 & & 0.284 & -0.286 & 6.06 & 2.29 & 4.24 & 0.0 & 4.24 & 1.75 & 12.86 & 909.90 & 0.239 \\
\hline${ }^{262} \mathrm{Sg}$ & & 1920.1 & 1909.3 & & 0.279 & -0.283 & 6.07 & 1.05 & 3.69 & 1.72 & 3.70 & 1.59 & 12.19 & 923.94 & 0.229 \\
\hline${ }^{271} \mathrm{Sg}$ & & 1976.5 & 1968.0 & & 0.254 & -0.188 & 6.12 & 1.39 & 4.12 & 0.0 & 3.45 & 0.93 & 7.71 & 981.87 & 0.212 \\
\hline${ }^{270} \mathrm{Bh}$ & & 1969.8 & 1958.7 & & 0.254 & -0.192 & 6.11 & 0.80 & 4.00 & 0.0 & 4.02 & 1.53 & 7.57 & 973.53 & 0.222 \\
\hline $\mathrm{Bh}$ & & 1980.8 & 1971.9 & & 0.244 & -0.187 & 6.12 & 0.0 & 4.21 & 0.0 & 4.62 & 1.07 & 7.82 & 35.27 & 0.221 \\
\hline${ }^{274} \mathrm{Bh}$ & & 1987.4 & 1984.7 & & 0.237 & $\mid-0.182$ & 6.13 & 0.0 & 4.25 & 0.0 & 3.16 & 0.52 & 8.20 & 996.40 & 0.192 \\
\hline${ }^{264} \mathrm{Hs}$ & & 1923.6 & 1912.8 & & 0.271 & -0.283 & 6.08 & 0.0 & 4.42 & 1.23 & 3.74 & 1.36 & 12.27 & 1927.62 & 0.229 \\
\hline${ }^{268} \mathrm{Hs}$ & & 1953.4 & 1941.3 & & 0.262 & -0.281 & 6.12 & 0.0 & 3.60 & 0.0 & 4.40 & 1.26 & 11.46 & 1957.28 & 0.230 \\
\hline${ }^{270} \mathrm{Hs}$ & & 1967.4 & 1955.2 & & 0.256 & $\mid-0.193$ & 6.11 & 0.0 & 4.17 & 0.0 & 4.17 & 1.76 & 7.69 & 1971.42 & 0.231 \\
\hline${ }^{272} \mathrm{Hs}$ & & 1978.9 & 1968.8 & & 0.248 & -0.188 & 6.12 & 0.0 & 4.40 & 0.0 & 4.40 & 1.48 & 7.84 & 1984.20 & 0.222 \\
\hline${ }^{275} \mathrm{Hs}$ & & 1995.8 & 1988.7 & & 0.233 & -0.180 & 6.14 & 0.0 & 4.39 & 0.0 & 3.84 & 0.78 & 8.42 & 000.78 & 0.183 \\
\hline $\mathrm{Mt}$ & & 1987.4 & 1978.7 & & 0.237 & -0.183 & 6.14 & 0.0 & 4.71 & 0.0 & 4.72 & 1.49 & 8.28 & 1991.77 & 0.222 \\
\hline $\mathrm{Mt}$ & & 1993.3 & 1985.5 & & 0.232 & -0.181 & 6.14 & 0.0 & 4.31 & 0.0 & 4.73 & 1.27 & 8.49 & 1998.36 & 0.212 \\
\hline $\mathrm{Mt}$ & & 1999.1 & 1992.2 & & 0.225 & -0.178 & 6.14 & 0.0 & 4.27 & 0.0 & 4.67 & 1.05 & 73 & 64 & 0.202 \\
\hline $\mathrm{Mt}$ & & 2010.6 & 2005.5 & & 0.212 & -0.173 & 5 & 0.0 & 4.38 & 0.0 & 4.49 & 0.70 & 9.30 & 32 & 0.136 \\
\hline Ds & & 1955.7 & 1945.5 & & 0.250 & -0.195 & 6.12 & 0.0 & 4.30 & 0.0 & 4.31 & 2.09 & .13 & 53 & 0.221 \\
\hline${ }^{279} \mathrm{Ds}$ & 2014.0 & 2011.7 & 2009.1 & 0.199 & 0.276 & -0 & 6.20 & 0 & 4. & 0 & 4.81 & 8 & 0 & & 0.127 \\
\hline${ }^{281} \mathrm{Ds}$ & 2019.8 & 2021.8 & 2022.3 & -0.030 & 0.340 & -0.166 & 6.24 & 16.96 & 22.10 & 0.0 & 4.44 & 0.88 & 10.34 & 91 & 0.108 \\
\hline${ }^{277} \mathrm{Rg}$ & & 1997.7 & 1991.3 & & 0.220 & -0.176 & 6.16 & 0.0 & 4.78 & 0.0 & 4.32 & 2.03 & 9.38 & 2003.04 & 0.193 \\
\hline $\mathrm{Rg}$ & & 2004.0 & 1998.4 & & 0.220 & -0. & 6.17 & 0.0 & 4.12 & 0.0 & 4.72 & 1.86 & .63 & 68 & 0.164 \\
\hline Rg & & 2007.1 & 2005.4 & & 0.288 & -0. & 6.21 & 0.0 & 4.09 & 0.8 & 4.24 & 1.68 & 0 & 73 & 0.164 \\
\hline $\mathrm{Rg}$ & & 2012.5 & 2012.3 & & 0.334 & -0. & 6.24 & 0.0 & 4.11 & 0.0 & 3 & 1.53 & 19 & 81 & 0.117 \\
\hline${ }^{281} \mathrm{Rg}$ & 2016.9 & 2018.4 & 2019.2 & -0.036 & 0.353 & -0. & 6.26 & 17.27 & 22.44 & 0.77 & 6 & 1.40 & 50 & .85 & 0.099 \\
\hline${ }^{2} \mathrm{Rg}$ & 2023.8 & 2024.3 & 2025.9 & -0.037 & 0.366 & & 6.27 & 17.06 & 20.14 & 0.62 & 4.61 & 1.29 & 79 & 82 & 0.099 \\
\hline 112 & 2020.4 & 2020.4 & 2022.3 & -0.030 & 0.370 & -0. & 6.28 & 17.85 & 23.20 & 0.0 & 4.75 & 1.94 & 07 & 2032.68 & 0.089 \\
\hline 112 & 2027.9 & 2028.2 & 2029.2 & -0.040 & 0.489 & -0.1 & 6.38 & 16.73 & 22.14 & 0.0 & 3.99 & 1.29 & 33 & .67 & 0.089 \\
\hline${ }^{284} 112$ & 2035.1 & 2035.2 & 2036.0 & -0.066 & 0.525 & -0.159 & 6.41 & 14.18 & 20.42 & 1.41 & 2.78 & 2.34 & 11.93 & 5.88 & 0.089 \\
\hline${ }^{285} 112$ & 2042.1 & 2041.6 & 2042.5 & -0.068 & 0.532 & -0.167 & 6.43 & 13.29 & 19.93 & 0.0 & 2.47 & 1.16 & 11.20 & 2051.61 & 0.089 \\
\hline${ }^{294} 112$ & 2099.4 & 2093.3 & 2098.9 & -0.004 & 0.559 & -0.005 & 6.50 & 3.07 & 15.17 & 0.0 & 5.74 & 0.22 & 11.86 & 2103.92 & 0.00 \\
\hline
\end{tabular}




\begin{tabular}{|c|c|c|c|c|c|c|c|c|c|c|c|c|c|c|c|}
\hline Nuclei & \multicolumn{3}{|c|}{$\mathrm{BE}$} & \multicolumn{3}{|c|}{$\beta_{2}$} & $r_{c h}$ & \multicolumn{2}{|c|}{ sph. } & \multicolumn{2}{|c|}{ prol. } & \multicolumn{2}{|c|}{ obl } & \multicolumn{2}{|c|}{ FRDM } \\
\hline & sph. & prol. & obl. & sph. & prol. & obl. & & $(\mathrm{DF})_{n}$ & $(\mathrm{DF})_{p}$ & $(\mathrm{DF})_{n}$ & $(\mathrm{DF})_{p}$ & $(\mathrm{DF})_{n}$ & $(\mathrm{DF})_{p}$ & $\overline{\mathrm{BE}}$ & $\overline{\beta_{2}}$ \\
\hline${ }^{32} 113$ & 2021.0 & 2021.1 & 2017.9 & -0.164 & 0.171 & -0.159 & 6.18 & 1.02 & 9.57 & 0.0 & 8.43 & 2.71 & 11.44 & 2027.20 & 0.071 \\
\hline${ }^{4} 113$ & 2031.0 & 2034.4 & 2032.3 & -0.002 & 0.158 & -0.154 & 6.18 & 18.47 & 24.09 & 0.0 & 8.61 & 2.37 & 11.93 & 2040.95 & 0.080 \\
\hline${ }^{5} 113$ & 2038.2 & 2041.1 & 2039.2 & -0.023 & 0.151 & -0.155 & 6.18 & 18.02 & 23.72 & 0.0 & 8.79 & 2.06 & 11.90 & 048.18 & 0.072 \\
\hline${ }^{6} 113$ & 2045.3 & 2047.7 & 2045.9 & -0.021 & 0.144 & 163 & 6.19 & 17.54 & 23.44 & 0.0 & 8.94 & 1.58 & 11.65 & 2054.22 & 0.072 \\
\hline${ }^{6} 114$ & 2041.4 & 2041.1 & 2042.0 & -0.012 & 0.555 & 150 & 6.46 & 18.59 & 24.53 & 0.0 & 0.0 & 2.63 & 12.55 & 2051.59 & -0.096 \\
\hline${ }^{7} 114$ & 2048.6 & 2047.7 & 2048.8 & -0.012 & 0.554 & 158 & 6.46 & 18.06 & 24.19 & 0.0 & 4.82 & 2.06 & 12.13 & 2057.65 & -0.078 \\
\hline${ }^{8} 114$ & 2055.8 & 2054.1 & 2055.5 & -0.024 & 0.547 & 65 & 6.46 & 16.76 & 23.18 & 0.0 & 4.63 & 2.14 & 12.09 & 2065.01 & 0.053 \\
\hline${ }^{9} 114$ & 2062.8 & 2060.3 & 2062.2 & 11 & 0.537 & 49 & 6.46 & 16.07 & 2 & 0.0 & 4.81 & 2.23 & 12.36 & 2071.04 & -0.052 \\
\hline 6114 & 2108.9 & 2102.3 & 2108.4 & 03 & 0.565 & & 6.53 & 4.05 & 1 & 0.0 & 4.32 & 1.68 & 68 & 3.22 & 0.00 \\
\hline${ }^{8} 114$ & 2121.1 & 2113.3 & 2120.8 & 00 & 0.566 & 12 & 6.54 & 0 & 1 & 0.0 & 4.72 & 0.0 & 10.74 & 123.30 & 0.00 \\
\hline 7115 & 2043.1 & 2044.9 & 2044.1 & -0.008 & 0.153 & 37 & 6.20 & 19.86 & 25.48 & 0.0 & 6.57 & 4.18 & 13.69 & 2052.72 & -0.096 \\
\hline${ }^{8} 115$ & 2050.5 & 2051.9 & 2051.2 & 005 & 145 & 37 & 6.20 & 9.24 & 09 & 0.0 & 7.07 & 3.66 & 13.38 & 2059.12 & -0.087 \\
\hline${ }^{9} 115$ & 2057.8 & 2058.8 & 2057.9 & -0.002 & 137 & 41 & 6.21 & .19 & 25.44 & 0.0 & 7.4 & 3.02 & 12.92 & 2066.45 & 0.053 \\
\hline 115 & 2064.9 & 2064.7 & 2065.2 & 0.000 & 117 & & 6.20 & 16.88 & 23.61 & 2.34 & 0.75 & 3.83 & 13.59 & 2072.59 & -0.079 \\
\hline 115 & 2071.9 & 2071.8 & 2072.4 & 0.000 & 0.064 & & 6.20 & 15.46 & 22.70 & 8.62 & 19.14 & 4.39 & 14.44 & 2079.83 & -0.061 \\
\hline 116 & 2059.5 & 2060.5 & 2060.3 & 0.002 & 0.134 & & 6.21 & 17.92 & 24.59 & 0.0 & 7.90 & 4.92 & 15.51 & 2068.76 & 0.072 \\
\hline${ }^{1} 116$ & 2066.7 & 2066.7 & 2067.5 & 0.002 & 0.110 & 08 & 6.21 & 16.61 & 23.78 & 0.3 & 11.81 & 5.56 & 15.29 & 2074.84 & 0.072 \\
\hline${ }^{2} 116$ & 2073.9 & 2074.1 & 74.7 & 0.002 & 0.062 & -0.097 & 6.21 & 15.32 & 22.96 & 10.18 & 19.89 & 5.37 & 15.45 & 2082.48 & -0.070 \\
\hline${ }^{33} 116$ & 2080.9 & 2081.1 & 81.8 & 0.002 & 0.052 & -0.092 & 6.21 & 13.55 & 21.98 & 10.76 & 2.35 & 4.07 & 14.79 & 2088.40 & -0.070 \\
\hline${ }^{33} 117$ & 2075.6 & 2074.3 & 076.4 & 0.039 & 0.536 & -0.099 & 6.50 & 13.64 & 22.17 & 0.0 & 0.0 & 4.57 & 55 & 2083.06 & -0.087 \\
\hline 94117 & 2082.8 & 2080.9 & 2083.6 & 0.043 & 0.538 & -0.095 & 6.51 & 12.18 & 21.27 & 0.0 & 0.0 & 2.99 & 77 & .22 & -0.087 \\
\hline${ }^{37} 117$ & 2103.6 & 2100.6 & 2103.3 & 0.031 & 0.561 & 53 & 6.55 & 8.27 & 36 & 0 & 0. & 0.0 & 36 & 9.27 & -0.008 \\
\hline${ }^{94} 118$ & 2076.9 & 2076.1 & 2077.7 & 0.055 & 0.548 & -0.102 & 6.52 & 9.32 & 19.71 & 0 . & 0.34 & 3.74 & 73 & 4.78 & -0.087 \\
\hline${ }^{97} 118$ & 2098.2 & 2096.6 & 2098.6 & -0.023 & 0.561 & -0.087 & 6.55 & 11.38 & 20.22 & 0. & 1.28 & 0.0 & 11.30 & 4.59 & -0.035 \\
\hline 292120 & 2046.4 & 2047.1 & 2047.3 & -0.006 & 0.530 & -0.125 & 6.50 & 26.49 & 31.54 & 0 . & 0.0 & 7.58 & 13 & 5.19 & -0.130 \\
\hline${ }^{3} 120$ & 2054.5 & 2054.9 & 2055.2 & -0.005 & 0.528 & -0.124 & 6.51 & 25.35 & 30.75 & 0.3 & 0.98 & 6.71 & 14.56 & 2062.42 & 0.089 \\
\hline${ }^{4} 120$ & 2134.9 & 2132.5 & 2132.7 & -0.008 & 0.558 & -0.074 & 6.59 & 0.0 & 8.36 & 0.0 & 3.62 & 0.0 & 10.01 & 2137.99 & 0.00 \\
\hline
\end{tabular}




\subsection{Shape and shape co-existence}

Nuclei have different binding energy corresponding to different shape configuration in the ground or excited states. In some cases, it so happens that the binding energy of two different configurations coincide or very close to each other known as shape co-existence $70 / 71 / 7273$. This phenomenon is more common in superheavy region, leads to complex structure of these nuclei which provides the information about the oscillation of nuclei between two or three existing shape by perturbing small energy. Here, in our studied nuclei, we find many such examples, where the ground and first excited binding energies are degenerated. Also, in several cases the nuclei have well defined three distinct solutions (oblate, spherical, prolate) with almost same energy which strongly indicates the nuclei may oscillate from oblate to spherical to prolate configuration.

For analyzing the result, we took a small binding energy difference around $\leq 2 \mathrm{MeV}$ to take care of shape co-existence for our calculations. Because of this small difference in binding energies between these two solutions, the ground state can be changed to the low-lying excited state or vice-versa by a small change in input parameters like pairing gaps etc. It is seen from Tables I and II the shape co-existence is observed for ${ }^{277,278,281,282} \mathrm{Rg},{ }^{282,283,284,285} 112,{ }^{286,287,288} 114$ ${ }^{294,297} 118$ and ${ }^{292,293} 120$ in SHF calculations and RMF also produces the shape coexistence for ${ }^{294} 112,{ }^{286} 113,{ }^{288,289,296,298} 114,{ }^{290,291} 115,{ }^{291,292} 116,{ }^{293,294,297} 117$, ${ }^{297} 118,{ }^{292,293} 120$. A shape co-existence of nuclei indicates that there is a competition among the different shapes to acquire the possible ground state for maximum stability and the final shape could be an admixture of these low-lying bands.

\subsection{Density and bubble structure}

The density of a nucleus has the gross information about the size, shape and distribution of nucleons. The density distribution from SHF(SkI4) and RMF(NL3*) for some of the selected nuclei are given in Figures 2 to 6 obtained for all the three solutions, i.e. spherical, prolate and oblate. Normally, the density at the center of nucleus has the maximum value and decays to zero at the surface. However, in some specific cases, e.g. for spherical solutions of ${ }^{285} 113,{ }^{294} 117,{ }^{292,293} 120$, this trend of density distribution shows an anomalous behavior, i.e. a dip at the center and a hump nearby to it following a slow decreasing in density to zero at the surface. This type of density distribution is known as bubble structure 74. The first possibility for existence of toroidal or bubble structure other than the spherical topological structure was suggested by Wheeler 74. The occurrence of bubble nuclei has been extensively studied by Wilson 75 and later by Siemens and Bethe 76 . Several models like independent particle model ${ }^{77}$ and Hartree Fock Model ${ }^{78}$ also investigated the possibility of low density region at the center of the nuclei. This structure is not confined to a particular region, but have the possibility for light to superheavy nuclei 79|80. The contribution of density at $r=0$ is offered by non zero wavefunction of $s$-states and depopulation of this level leads to the depletion of central 
Table 2. Same as Table I with RMF(NL3*) model.

\begin{tabular}{|c|c|c|c|c|c|c|c|c|c|c|c|c|c|}
\hline Nuclei & \multicolumn{3}{|c|}{$\mathrm{BE}$} & \multicolumn{3}{|c|}{$\overline{\beta_{2}}$} & $r_{c h .}$ & \multicolumn{2}{|c|}{ sph. } & \multicolumn{2}{|c|}{ prol. } & \multicolumn{2}{|c|}{ obl. } \\
\hline & sph. & prol. & obl. & sph. & prol. & obl. & & $(\mathrm{DF})_{n}$ & $(\mathrm{DF})_{p}$ & $(\mathrm{DF})_{n}$ & $(\mathrm{DF})_{p}$ & $(\mathrm{DF})_{n}$ & $(\mathrm{DF})_{p}$ \\
\hline $\mathrm{Db}$ & 1941.5 & 1955.9 & 946.2 & 0.002 & 0.273 & -0.308 & 29 & 19.57 & 18.00 & 1.22 & 2.95 & 5.22 & 20.05 \\
\hline${ }^{267} \mathrm{Db}$ & 1947.9 & 1.6 & 2.1 & 02 & 269 & 07 & & 19.79 & & & 1. & & 67 \\
\hline${ }^{268} \mathrm{Db}$ & 1954.3 & 1966.7 & 7.9 & 001 & 0.267 & 06 & & 19.98 & 9 & 92 & 2.92 & & 32 \\
\hline${ }^{9} \mathrm{Db}$ & 1960.4 & 1971.4 & 1963.6 & 001 & 0.263 & -0.306 & 43 & 20.20 & 2 & 87 & 1.55 & 19 & .66 \\
\hline${ }^{0} \mathrm{Db}$ & 1966.5 & 1976.0 & 1969.1 & 002 & 0.256 & -0.306 & 147 & 20.40 & 18.78 & 0.87 & 1.23 & 1.27 & 1.14 \\
\hline${ }^{258} \mathrm{Sg}$ & 1883.6 & 1899.6 & 1889.5 & 004 & 0.291 & -0.301 & 091 & 17.28 & 16.26 & 6.26 & 4.43 & 9.54 & 8.95 \\
\hline${ }^{260} \mathrm{Sg}$ & 1898.1 & 1913.9 & 1903.9 & 0.002 & 0.285 & -0.301 & 102 & 18.04 & 16.86 & 6.18 & 4.46 & 7.35 & 9.66 \\
\hline${ }^{262} \mathrm{Sg}$ & 1912.1 & 1927.7 & 1917.7 & 002 & 0.281 & -0.305 & 113 & 18.68 & 17.38 & 5.32 & 4.33 & .69 & 19.37 \\
\hline${ }^{271} \mathrm{Sg}$ & & 1980.9 & & 02 & 0.254 & & & 20.68 & & & 3.48 & & \\
\hline${ }^{270} \mathrm{Bh}$ & & 1975.3 & 1965.9 & 0.002 & 0.262 & 11 & & 0.42 & 19.16 & 0.60 & 4.21 & 58 & .16 \\
\hline${ }^{272} \mathrm{Bh}$ & 1975.9 & 1985.4 & 7.8 & 002 & 0.244 & & & 20.87 & 19.69 & & .11 & 89 & .86 \\
\hline${ }^{274} \mathrm{Bh}$ & 1995.9 & 1990.8 & 1989.3 & 205 & 0.216 & -0.319 & 62 & 1.00 & 6.77 & 0.54 & 0.26 & .99 & 2.15 \\
\hline${ }^{4} \mathrm{Hs}$ & & 1932.1 & 1922.6 & & 0.269 & -0.311 & 130 & 5.72 & 7.62 & 6.06 & 4.62 & .28 & 9.75 \\
\hline $3 \mathrm{Hs}$ & & 1959.9 & 1950.1 & & 0.265 & -0.315 & 52 & 1.98 & 5.76 & 1.86 & 3.08 & 3.64 & 9.94 \\
\hline $\mathrm{Hs}$ & & 1972.9 & 1963.1 & & 0.260 & -0.314 & 61 & 0.78 & 5.24 & 0.88 & 4.85 & .34 & .10 \\
\hline${ }^{272} \mathrm{Hs}$ & & 1984.1 & 1975.6 & & 0.249 & -0.313 & 69 & 0.43 & 5.29 & 0.99 & 4.78 & 55 & 0.51 \\
\hline${ }^{275} \mathrm{Hs}$ & & 2000.6 & & & 203 & & & 1.19 & 7.66 & 1.40 & 8 . & 9 & 2.19 \\
\hline${ }^{274} \mathrm{Mt}$ & & 1993.4 & 5.1 & & 16 & -0. & & 1. & 9 & 1.49 & 6 & 9 & 1.46 \\
\hline${ }^{275} \mathrm{Mt}$ & & 9.1 & .3 & & 07 & & & 1.22 & 0 & 47 & 0 . & 9 & 2.16 \\
\hline${ }^{276} \mathrm{Mt}$ & & 2004.7 & .4 & & 0.199 & & & 1.47 & 6 & 43 & 0 & 6 & 22.77 \\
\hline${ }^{8} \mathrm{Mt}$ & 2015.8 & 2013.6 & 2009.4 & 0.185 & 0.373 & -0.330 & & 1.39 & 8.30 & 1.06 & 3.68 & 53 & 23.77 \\
\hline Ds & & 1963.9 & 1954.9 & & 0.251 & -0.319 & & 2.84 & 7.77 & 38 & 8.09 & 35 & 20.93 \\
\hline Ds & 2020.0 & 2017.4 & 2013.3 & 0.182 & 0.384 & -0.336 & 96 & 1.40 & 8.57 & 2.63 & & 81 & 4.27 \\
\hline Ds & 2031.2 & 2029.2 & 2025.4 & 0.168 & 0.476 & -0.2 & & 1.65 & & 4.93 & 7.06 & 76 & 4.47 \\
\hline${ }^{277} \mathrm{Rg}$ & 005.6 & 002.7 & 1997.8 & 0.199 & 0.341 & & & 1.39 & & 2.16 & & .15 & 6.13 \\
\hline${ }^{278} \mathrm{Rg}$ & & .8 & & 0.191 & 0. & & & 1.41 & & 2 & & 8 & .22 \\
\hline${ }^{9} \mathrm{Rg}$ & 7.9 & & 7 & 0. & & & & 1 . & & 2 & & 18 & 26 \\
\hline${ }^{0} \mathrm{Rg}$ & 2023.9 & 6 & .0 & 79 & 35 & & & 1 & & 44 & 2 & 6 & .27 \\
\hline${ }^{1} \mathrm{Rg}$ & 9.7 & .2 & .3 & 73 & 03 & & & & & 42 & & 9 & 22 \\
\hline $\operatorname{Rg}$ & 5.4 & 2033.3 & .4 & 0.166 & 0.497 & -0.339 & 12 & 1.59 & 4 & 86 & 8.17 & 7.05 & 5.23 \\
\hline 112 & & 030.8 & 2026.8 & 0.171 & 0.515 & -0.346 & 19 & & 9.06 & 79 & 8. & 3 & 25.95 \\
\hline 112 & 039.2 & 2037.2 & 2033.2 & 0.165 & 0.508 & -0.343 & 6.222 & 1. & 9 . & 22 & 7 . & 7.29 & 25.92 \\
\hline 112 & 041.3 & 043.4 & 2039.3 & 0.002 & 0.501 & -0.340 & 6.436 & 23.96 & 22.74 & 5 & & 7.25 & 25.95 \\
\hline 112 & & & & & & & & 23.56 & 22.59 & & & 7.15 & 26.04 \\
\hline 112 & & .3 & & 0 & & & & 8.94 & 17.06 & 6 & & .89 & 17.18 \\
\hline 113 & & & & & & & & 2 & .04 & 2.27 & 7 . & 45 & 41 \\
\hline 113 & & & & & & & & & & & & 46 & 42 \\
\hline 113 & & & & 0.001 & & & & & & 2 & 10 & 46 & 48 \\
\hline 113 & 1.4 & 20 & & 0.002 & 0.502 & -0.148 & 54 & & & 4 & & 97 & 14.25 \\
\hline 14 & 2048.6 & 2051.6 & 2046.0 & 0.001 & 0.158 & -0.347 & 6.246 & 25 & $2:$ & 1.73 & & 7.57 & 82 \\
\hline 114 & 054.9 & 2057.6 & 2054.8 & 0.001 & 0.151 & -0.173 & & & 23.22 & 9 & & 8 & 80 \\
\hline 114 & & 063.1 & & & & & & 24.10 & 22.91 & 6 & & 7.17 & \\
\hline 14 & & 9.1 & & & & & & 23.24 & 22.60 & 4.66 & 7 . & .56 & 98 \\
\hline 14 & & & & & & & & 10.22 & & 5.37 & 7 . & 3.08 & 19 \\
\hline 14 & 2118.0 & 21 & 21 & & & & & & 94 & & & .02 & 45 \\
\hline 115 & 2051.7 & 2053.8 & 2051.5 & & & & & & & & & 34 & 44 \\
\hline 115 & 2058.2 & 2060.1 & 2057.9 & 0.001 & & & & 25.52 & & & & & 15.41 \\
\hline 115 & 2064.5 & 2066.1 & 2064.5 & 0.002 & & & & 24.84 & & 2.69 & 10.65 & 7.24 & 15.47 \\
\hline 115 & 2070.6 & 2072.4 & 2070.7 & 0.002 & 0.515 & -0.195 & & 23.96 & 22.98 & 4.05 & 3.20 & 6.64 & 15.61 \\
\hline${ }^{91} 115$ & 2077.3 & 2078.5 & 2076.8 & 0.073 & 0.518 & -0.213 & .507 & 13.28 & 18.94 & 3.92 & 5.22 & 6.39 & 16.10 \\
\hline
\end{tabular}

continue... 


\begin{tabular}{|c|c|c|c|c|c|c|c|c|c|c|c|c|c|}
\hline Vuclei & \multicolumn{3}{|c|}{$\mathrm{BE}$} & \multicolumn{3}{|c|}{$\beta_{2}$} & ch & \multicolumn{2}{|c|}{ sph. } & \multicolumn{2}{|c|}{ prol. } & \multicolumn{2}{|c|}{ obl. } \\
\hline & sph. & prol. & obl. & sph. & prol. & obl. & & $(\mathrm{DF})_{n}$ & $(\mathrm{DF})_{p}$ & $(\overline{D F})_{n}$ & $(\mathrm{DF})_{p}$ & $(\mathrm{DF})_{n}$ & $(\mathrm{DF})_{p}$ \\
\hline${ }^{0} 116$ & 2067.5 & 2068.1 & 2067.2 & 0.012 & 0.144 & -0.187 & 6.271 & 25.22 & 23.65 & 2.20 & 10.26 & 7.53 & 16.11 \\
\hline${ }^{1} 116$ & 2073.9 & 2075.3 & 2073.7 & 0.044 & 0.522 & -0.194 & .561 & 20.61 & 22.01 & 3.40 & 3.65 & 6.92 & 16.17 \\
\hline${ }^{2} 116$ & 2080.3 & 2081.7 & 2079.9 & 0.052 & 0.525 & -0.212 & .524 & 17.45 & 19.62 & 3.31 & 3.41 & 6.82 & 16.53 \\
\hline 116 & & 2 & 2 & 0. & 0.529 & -0.2 & 32 & 10 & 2 & 2 & 9 & 6.81 & 72 \\
\hline 117 & & .4 & 2 & 0 & 0.531 & -0.3 & 0 & 24 & 2 & 5 & 2.06 & 33 & 12 \\
\hline${ }^{4} 117$ & 4 & 0.7 & 20 & & 35 & 5.3 & 50 & 7 & 2 & 3.00 & 2.40 & 7.77 & 33 \\
\hline${ }^{7} 117$ & 2 & 8.9 & 2 & & 50 & 52 & & 05 & & 3.75 & 3.33 & 7.62 & .81 \\
\hline${ }^{4} 118$ & 21 & 2095.8 & 2094.2 & & .529 & -0 & & 15.06 & & 4.20 & 5.02 & 5.79 & 15.99 \\
\hline${ }^{7} 118$ & 21 & 2112.2 & 2109.2 & & 0.567 & -0.8 & & 10.88 & & 4. & 5.64 & 16 & 15.53 \\
\hline 2120 & 2061.5 & 2060.8 & 2057.9 & 0.000 & 0.547 & -0.392 & 1 & 28.92 & & 3. & 1.47 & .51 & 21.78 \\
\hline 3120 & 2068.7 & 2068.3 & 2065.1 & 0.000 & 0.542 & -0.388 & 6 & 28.56 & & 3.09 & 1.48 & 3.75 & 29.11 \\
\hline${ }^{4} 120$ & 2139.3 & 2142.4 & 2137.4 & 0.000 & 0.592 & -0.369 & .678 & 3.68 & 16.00 & 3.44 & 0.81 & 8.33 & 29.69 \\
\hline
\end{tabular}

density and formed a bubble like structure 80 . The inversion of $s$-states to other higher states may also be the possibility of depletion of central density and can be interpreted as $s$ - $d$ orbital inversion 8182 .

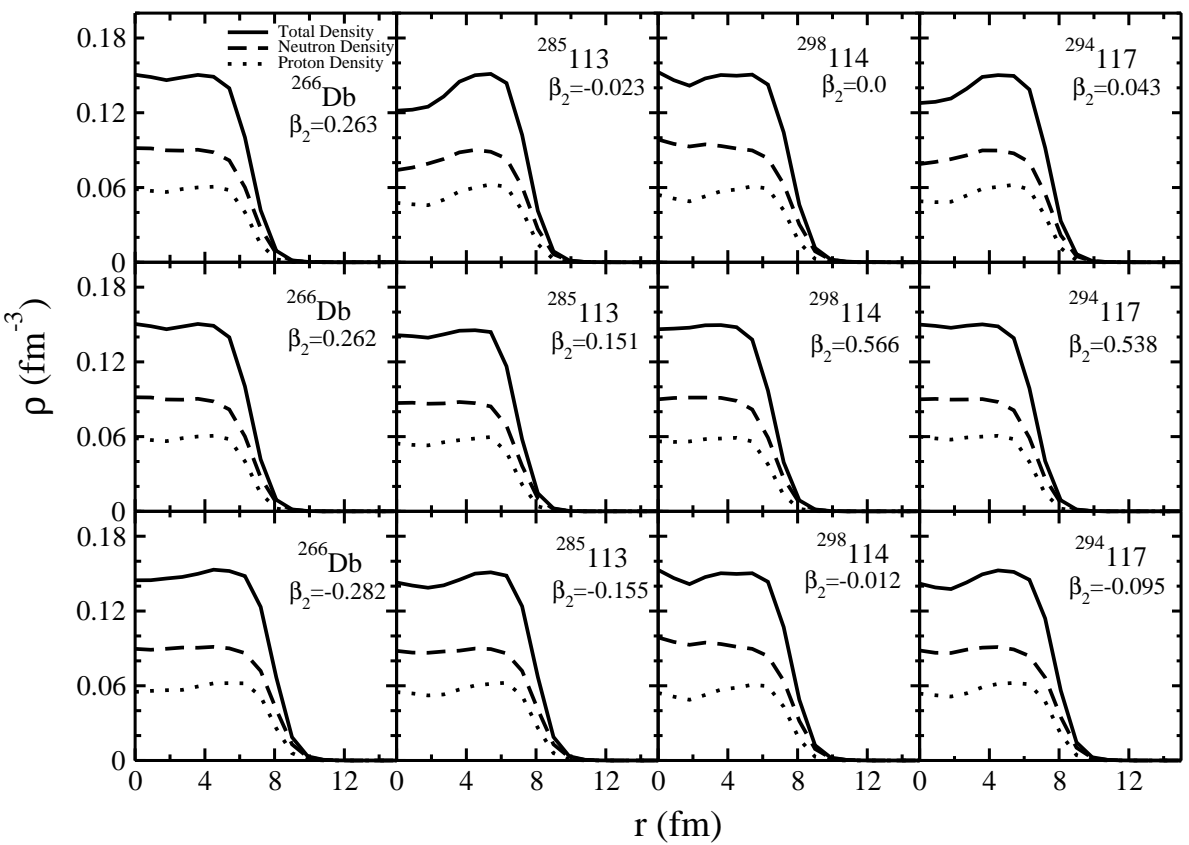

Fig. 2. The neutron, proton and total matter density distribution for some of the selected superheavy nuclei using SHF(SkI4). 
Table 3. The calculated $Q_{\alpha}$ and life time $T_{\alpha}$ using $\mathrm{SHF}(\mathrm{SkI} 4)$ and RMF(NL3*) are compared with FRDM and experimental data, wherever available.

\begin{tabular}{|c|c|c|c|c|c|c|c|c|}
\hline Nuclei & \multicolumn{4}{|c|}{$Q_{\alpha}(\mathrm{MeV})$} & \multicolumn{4}{|c|}{$T_{\alpha}$} \\
\hline & SHF & RMF & FRDM & Expt. & SHF & RMF & FRDM & Expt. \\
\hline${ }^{266} \mathrm{Db}$ & 7.64 & 7.17 & 7.36 & & $1.03 \times 10^{6} \mathrm{~s}$ & $8.87 \times 10^{7} \mathrm{~s}$ & $10^{7.15} \mathrm{~s}$ & \\
\hline${ }^{267} \mathrm{Db}$ & 7.41 & 7.15 & 7.09 & & $3.94 \times 10^{6} \mathrm{~s}$ & $4.84 \times 10^{7} \mathrm{~s}$ & $10^{7.96} \mathrm{~s}$ & \\
\hline${ }^{268} \mathrm{Db}$ & 8.01 & 7.49 & 7.19 & & $40673.0 \mathrm{~s}$ & $4.23 \times 10^{6} \mathrm{~s}$ & $10^{7.87} \mathrm{~s}$ & \\
\hline${ }^{269} \mathrm{Db}$ & 8.41 & 7.98 & 7.59 & & $717.39 \mathrm{~s}$ & $2.32 \times 10^{4} \mathrm{~s}$ & $10^{6.95} \mathrm{~s}$ & \\
\hline${ }^{270} \mathrm{Db}$ & 8.38 & 8.19 & 7.84 & $8.80 \pm 0.1$ & $1995.7 \mathrm{~s}$ & $9073.49 \mathrm{~s}$ & $10^{5.25} \mathrm{~s}$ & $1.3 \mathrm{~min}$ \\
\hline${ }^{258} \mathrm{Sg}$ & 10.02 & 9.48 & 9.45 & & $4.05 \mathrm{~ms}$ & $0.13 \mathrm{~s}$ & $10^{-0.83} \mathrm{~s}$ & \\
\hline${ }^{260} \mathrm{Sg}$ & 9.49 & 9.33 & 9.93 & & $0.12 \mathrm{~s}$ & $0.34 \mathrm{~s}$ & $10^{-2.15} \mathrm{~s}$ & \\
\hline${ }^{262} \mathrm{Sg}$ & 8.80 & 9.04 & 9.61 & & $14.42 \mathrm{~s}$ & $2.63 \mathrm{~s}$ & $10^{-1.26} \mathrm{~s}$ & \\
\hline${ }^{271} \mathrm{Sg}$ & 8.84 & 8.88 & 8.40 & $8.54 \pm 0.08$ & $125.06 \mathrm{~s}$ & $95.39 \mathrm{~s}$ & $10^{3.55} \mathrm{~s}$ & $1.9_{-0.6}^{+2.4} \mathrm{~s}$ \\
\hline${ }^{270} \mathrm{Bh}$ & 8.80 & 8.92 & 8.18 & $8.93 \pm 0.08$ & $427.56 \mathrm{~s}$ & $173.58 \mathrm{~s}$ & $10^{4.76} \mathrm{~s}$ & $87.98 \mathrm{~s}$ \\
\hline${ }^{272} \mathrm{Bh}$ & 9.20 & 9.61 & 8.88 & 9.01 & $23.83 \mathrm{~s}$ & $1.49 \mathrm{~s}$ & $10^{2.36} \mathrm{~s}$ & $11.8 \mathrm{~s}$ \\
\hline${ }^{274} \mathrm{Bh}$ & 8.60 & 8.34 & 8.71 & $8.80 \pm 0.1$ & $1953.31 \mathrm{~s}$ & $14779.07 \mathrm{~s}$ & $10^{2.93} \mathrm{~s}$ & $1.3 \mathrm{~min}$ \\
\hline${ }^{264} \mathrm{Hs}$ & 10.20 & 10.06 & 10.57 & & $5.94 \mathrm{~ms}$ & $14.3 \mathrm{~ms}$ & $10^{-3.18} \mathrm{~s}$ & \\
\hline${ }^{268} \mathrm{Hs}$ & 9.12 & 9.59 & 9.00 & & $7.16 \mathrm{~s}$ & $28.2 \mathrm{~ms}$ & $10^{1.24} \mathrm{~s}$ & \\
\hline${ }^{270} \mathrm{Hs}$ & 8.58 & 9.44 & 8.69 & & $406.49 \mathrm{~s}$ & $79.2 \mathrm{~ms}$ & $10^{2.23} \mathrm{~s}$ & \\
\hline${ }^{272} \mathrm{Hs}$ & 9.98 & 10.08 & 9.20 & & $22.95 \mathrm{~ms}$ & $12.1 \mathrm{~ms}$ & $10^{0.61} \mathrm{~s}$ & \\
\hline${ }^{275} \mathrm{Hs}$ & 9.00 & 8.75 & 9.39 & $9.30 \pm 0.06$ & $198.15 \mathrm{~s}$ & $1255.21 \mathrm{~s}$ & $10^{1.09} \mathrm{~s}$ & $0.19_{-0.07}^{+0.22} \mathrm{~s}$ \\
\hline${ }^{274} \mathrm{Mt}$ & 10.70 & 10.13 & 10.06 & $10.02 \pm 1.08$ & $8.48 \mathrm{~ms}$ & $249.99 \mathrm{~ms}$ & $10^{-0.41} \mathrm{~s}$ & $472.6 \mathrm{~ms}$ \\
\hline${ }^{275} \mathrm{Mt}$ & 10.34 & 9.60 & 10.06 & 9.67 & $0.03 \mathrm{~s}$ & $3.30 \mathrm{~s}$ & $10^{-0.77} \mathrm{~s}$ & $0.98 \mathrm{~s}$ \\
\hline${ }^{276} \mathrm{Mt}$ & 10.00 & 8.92 & 9.93 & 9.71 & $0.55 \mathrm{~s}$ & $906.38 \mathrm{~s}$ & $10^{-0.05} \mathrm{~s}$ & $0.72 \mathrm{~s}$ \\
\hline${ }^{278} \mathrm{Mt}$ & 9.30 & 8.45 & 9.37 & $9.55 \pm 0.19$ & $57.68 \mathrm{~s}$ & $34927.55 \mathrm{~s}$ & $10^{1.54} \mathrm{~s}$ & $11.0 \mathrm{~s}$ \\
\hline${ }^{270} \mathrm{Ds}$ & 11.38 & 10.41 & 10.31 & & $3.19 \times 10^{5} \mathrm{~s}$ & $7.1 \mathrm{~ms}$ & $10^{-1.89} \mathrm{~s}$ & \\
\hline${ }^{279} \mathrm{Ds}$ & 10.10 & 8.85 & 9.68 & $9.70 \pm 0.06$ & $55.67 \mathrm{~ms}$ & $3047.07 \mathrm{~s}$ & $10^{0.91} \mathrm{~s}$ & $0.2_{-0.04}^{+0.05} \mathrm{~s}$ \\
\hline${ }^{281} \mathrm{Ds}$ & 13.34 & 8.37 & 8.55 & 8.83 & $4.21 \times 10^{-8} \mathrm{~s}$ & $1.39 \times 10^{5} \mathrm{~s}$ & $10^{4.52} \mathrm{~s}$ & $1.6 \mathrm{~min}$ \\
\hline${ }^{277} \mathrm{Rg}$ & 12.04 & 10.04 & 11.50 & & $1.31 \times 10^{-5} \mathrm{~s}$ & $0.865 \mathrm{~s}$ & $10^{-3.72} \mathrm{~s}$ & \\
\hline${ }^{278} \mathrm{Rg}$ & 11.70 & 9.89 & 11.39 & $10.69 \pm 0.08$ & $0.15 \mathrm{~s}$ & $4.93 \mathrm{~s}$ & $10^{3.11_{\mathrm{s}}}$ & $6.2 \mathrm{~ms}$ \\
\hline${ }^{279} \mathrm{Rg}$ & 11.30 & 9.52 & 10.92 & 10.37 & $0.56 \mathrm{~s}$ & $27.53 \mathrm{~s}$ & $10^{-2.36} \mathrm{~s}$ & $0.17 \mathrm{~s}$ \\
\hline${ }^{280} \mathrm{Rg}$ & 10.80 & 9.20 & 10.13 & 9.75 & $19.59 \mathrm{~s}$ & 557.61s & $10^{0.03} \mathrm{~s}$ & $5.1 \mathrm{~s}$ \\
\hline${ }^{281} \mathrm{Rg}$ & 14.77 & 12.99 & 9.37 & & $1.55 \times 10^{-10} \mathrm{~s}$ & $1.64 \times 10^{-7} \mathrm{~s}$ & $10^{1.90} \mathrm{~s}$ & \\
\hline${ }^{282} \mathrm{Rg}$ & 14.60 & 8.76 & 8.79 & $9.00 \pm 0.1$ & $6.29 \times 10^{-10} \mathrm{~s}$ & $15526.06 \mathrm{~s}$ & $10^{4.10} \mathrm{~s}$ & $0.74 \mathrm{~s}$ \\
\hline${ }^{282} 112$ & 15.87 & 9.37 & 9.42 & & $1.04 \times 10^{-12} \mathrm{~S}$ & $29.46 \mathrm{~s}$ & $10^{1.30} \mathrm{~s}$ & \\
\hline${ }^{283} 112$ & 14.10 & 9.09 & 9.01 & $9.34 \pm 0.06$ & $6.32 \times 10^{-9} \mathrm{~s}$ & $2666.54 \mathrm{~s}$ & $10^{3.66} \mathrm{~s}$ & $3.8_{-0.7}^{+1.2} \mathrm{~S}$ \\
\hline${ }^{284} 112$ & 11.48 & 10.57 & 8.69 & $9.17 \pm 0.05$ & $7.15 \times 10^{-5} \mathrm{~s}$ & $0.012 \mathrm{~s}$ & $10^{3.67} \mathrm{~s}$ & $9.8_{-3.8}^{+18} \mathrm{~s}$ \\
\hline${ }^{285} 112$ & 8.00 & 9.98 & 8.59 & 9.15 & $1.97 \times 10^{7} \mathrm{~s}$ & $5.44 \mathrm{~s}$ & $10^{5.09} \mathrm{~s}$ & $29 \mathrm{~s}$ \\
\hline 94112 & 6.12 & 7.71 & 7.53 & & $1.69 \times 10^{15} \mathrm{~s}$ & $2.46 \times 10^{7} \mathrm{~s}$ & $10^{8.15} \mathrm{~s}$ & \\
\hline 82113 & 11.12 & 9.89 & 9.78 & $10.62 \pm 0.08$ & $8.20 \mathrm{~ms}$ & $21.28 \mathrm{~s}$ & $10^{1.66} \mathrm{~s}$ & $88.9 \mathrm{~ms}$ \\
\hline${ }^{84} 113$ & 10.50 & 9.68 & 9.15 & $9.97 \pm 0.05$ & $46.84 \mathrm{~ms}$ & $94.43 \mathrm{~s}$ & $10^{3.61} \mathrm{~s}$ & $1.36 \mathrm{~s}$ \\
\hline 285113 & 5.60 & 9.45 & 8.97 & $9.48 \pm 0.11$ & $5.74 \times 10^{19} \mathrm{~s}$ & $203.59 \mathrm{~s}$ & $10^{3.86} \mathrm{~s}$ & $7.9 \mathrm{~s}$ \\
\hline${ }^{86} 113$ & 4.00 & 10.34 & 8.90 & $9.63 \pm 0.1$ & $9.55 \times 10^{33} \mathrm{~s}$ & $1.27 \mathrm{~s}$ & $10^{4.44} \mathrm{~s}$ & $28.3 \mathrm{~s}$ \\
\hline${ }^{286} 114$ & 7.60 & 10.05 & 9.39 & $10.19 \pm 0.06$ & $4.57 \times 10^{8} \mathrm{~s}$ & $1.26 \mathrm{~s}$ & $10^{2.08} \mathrm{~s}$ & $0.13_{-0.01}^{+0.01} \mathrm{~s}$ \\
\hline${ }^{287} 114$ & 8.80 & 9.94 & 9.31 & 9.54 & $1.23 \times 10^{5} \mathrm{~s}$ & $29.91 \mathrm{~s}$ & $10^{3.39} \mathrm{~s}$ & $3.8 \mathrm{~s}$ \\
\hline${ }^{288} 114$ & 7.70 & 8.61 & 9.16 & $9.84 \pm 0.05$ & $1.71 \times 10^{8} \mathrm{~s}$ & $4.83 \times 10^{4} \mathrm{~s}$ & $10^{2.80} \mathrm{~s}$ & $1.9_{-0.8}^{+3.8} \mathrm{~s}$ \\
\hline${ }^{289} 114$ & 7.60 & 8.66 & 8.87 & 9.82 & $5.33 \times 10^{9} \mathrm{~s}$ & $3.67 \times 10^{5} \mathrm{~s}$ & $10^{4.86} \mathrm{~s}$ & $2.6 \mathrm{~s}$ \\
\hline${ }^{296} 114$ & 6.75 & 8.32 & 8.68 & & $4.68 \times 10^{12} \mathrm{~s}$ & $5.864 \times 10^{5} \mathrm{~s}$ & $10^{4.43} \mathrm{~s}$ & \\
\hline 298114 & 15.6 & 7.91 & 8.91 & & $6.92 \times 10^{-12} \mathrm{~S}$ & $2.389 \times 10^{7} \mathrm{~s}$ & $10^{3.63} \mathrm{~s}$ & \\
\hline${ }^{287} 115$ & 7.09 & 11.01 & 10.25 & 10.59 & $1.49 \times 10^{12} \mathrm{~s}$ & $42.8 \mathrm{~ms}$ & $10^{0.63} \mathrm{~s}$ & $32 \mathrm{~ms}$ \\
\hline 288115 & 10.80 & 10.75 & 10.12 & 10.48 & $318.88 \mathrm{~ms}$ & $426.6 \mathrm{~ms}$ & $10^{1.33} \mathrm{~s}$ & $250 \mathrm{~ms}$ \\
\hline${ }^{289} 115$ & 10.60 & 10.74 & 10.03 & $10.31 \pm 0.09$ & $482.31 \mathrm{~ms}$ & $204.84 \mathrm{~ms}$ & $10^{1.26} \mathrm{~s}$ & $320 \mathrm{~ms}$ \\
\hline${ }^{290} 115$ & 12.20 & 9.23 & 9.92 & $9.95 \pm 0.4$ & $0.165 \mathrm{~s}$ & $11050.16 \mathrm{~s}$ & $10^{1.90} \mathrm{~s}$ & $0.023 \mathrm{~s}$ \\
\hline${ }^{91} 115$ & 8.86 & 10.15 & 9.66 & & $87783.45 \mathrm{~s}$ & $8.45 \mathrm{~s}$ & $10^{2.34} \mathrm{~s}$ & \\
\hline
\end{tabular}

continue... 


\begin{tabular}{|c|c|c|c|c|c|c|c|c|}
\hline Nuclei & \multicolumn{4}{|c|}{$Q_{\alpha}(\mathrm{MeV})$} & \multicolumn{4}{|c|}{$T_{\alpha}$} \\
\hline & $\mathrm{SHF}$ & RMF & FRDM & Expt. & SHF & RMF & FRDM & Expt. \\
\hline${ }^{290} 116$ & 8.90 & 11.76 & 11.12 & $0.84 \pm 0.08$ & $24443.12 \mathrm{~s}$ & $0.23 \mathrm{~ms}$ & $10^{-2.12} \mathrm{~s}$ & $7.1_{-1.7}^{+3.2} \mathrm{~s}$ \\
\hline${ }^{291} 116$ & 8.50 & 10.58 & 11.11 & $10.74 \pm 0.07$ & $7.77 \times 10^{6} \mathrm{~s}$ & $2.25 \mathrm{~s}$ & $10^{-1.03} \mathrm{~s}$ & $18_{-6}^{+22} \mathrm{~ms}$ \\
\hline${ }^{292} 116$ & 9.40 & 9.689 & 10.82 & $10.71 \pm 0.15$ & $528.65 \mathrm{~s}$ & $1 \mathrm{~s}$ & $10^{-1.37} \mathrm{~s}$ & $33_{-15}^{+155} \mathrm{~ms}$ \\
\hline${ }^{293} 116$ & 9.30 & 979 & 10.94 & $10.67 \pm 0.06$ & $12923.99 \mathrm{~s}$ & $825.50 \mathrm{~s}$ & $10^{-0.59} \mathrm{~s}$ & $53_{-19}^{+62} \mathrm{~m}$ \\
\hline${ }^{3} 117$ & 10.70 & 989 & 11.68 & 11.03 & $1.07 \mathrm{~s}$ & $104.71 \mathrm{~s}$ & $10^{-2.40} \mathrm{~s}$ & $21 \mathrm{~ms}$ \\
\hline${ }^{4} 117$ & 9.40 & 10.00 & 11.67 & $10.81 \pm 0.1$ & 15027.71s & $213.62 \mathrm{~s}$ & $.05 \mathrm{~S}$ & $112 \mathrm{~ms}$ \\
\hline 117 & 10.74 & 9.52 & 11.74 & & $0.844 \mathrm{~s}$ & $2869.78 \mathrm{~s}$ & $10^{-2.55} \mathrm{~s}$ & \\
\hline 118 & 11.10 & 9.60 & 12.28 & 10.84 & $33.36 \mathrm{~ms}$ & $553.44 \mathrm{~s}$ & $10^{-4.24} \mathrm{~s}$ & $7.1 \mathrm{~ms}$ \\
\hline 718 & 4.40 & 9.95 & 12.10 & & $5.13 \times 10^{32} \mathrm{~s}$ & $555.59 \mathrm{~s}$ & $10^{-2.79} \mathrm{~S}$ & \\
\hline 120 & 12.42 & 10.37 & 13.89 & & $9.68 \times 10^{-5} \mathrm{~s}$ & $12.30 \mathrm{~s}$ & & \\
\hline 120 & 12.24 & 11.05 & 13.69 & & $2.81 \mathrm{~ms}$ & $2.09 \mathrm{~s}$ & & \\
\hline 120 & 12.07 & 10.19 & 13.82 & & $0.58 \mathrm{~ms}$ & $42.53 \mathrm{~s}$ & $10^{-}$ & \\
\hline
\end{tabular}

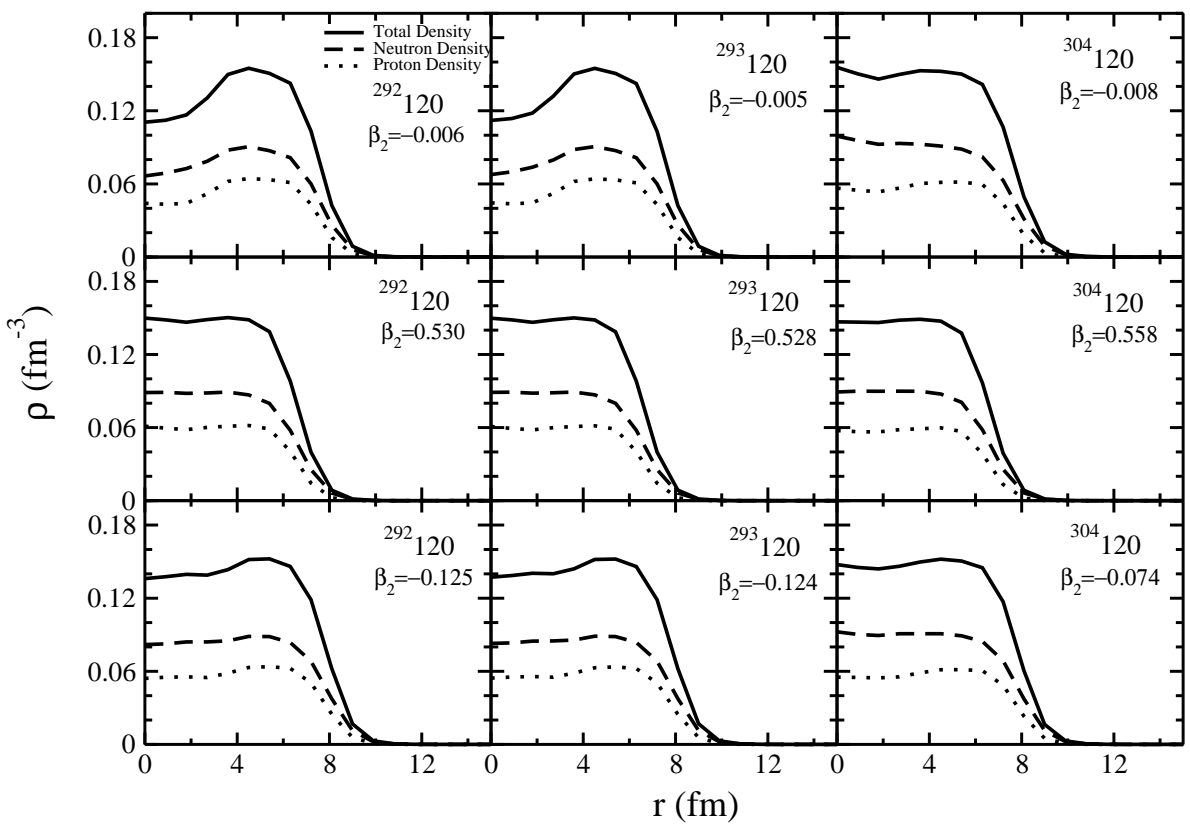

Fig. 3. Same as Fig. 3, but for ${ }^{292,293,304} 120$ with SHF(SkI4).

The bubble effect can be realized quantitatively by calculating depletion fraction for the neutrons as well as protons using the relation 80

$$
(D . F .)_{\alpha}=\frac{\left(\rho_{\max }\right)_{\alpha}-\left(\rho_{\text {cen }}\right)_{\alpha}}{\left(\rho_{\max }\right)_{\alpha}} \times 100,
$$

where $\rho_{\max }, \rho_{\text {cen }}$ represent the maximum and central density respectively and $\alpha$ denotes neutron or proton. The calculated values of depletion fraction for neutron 


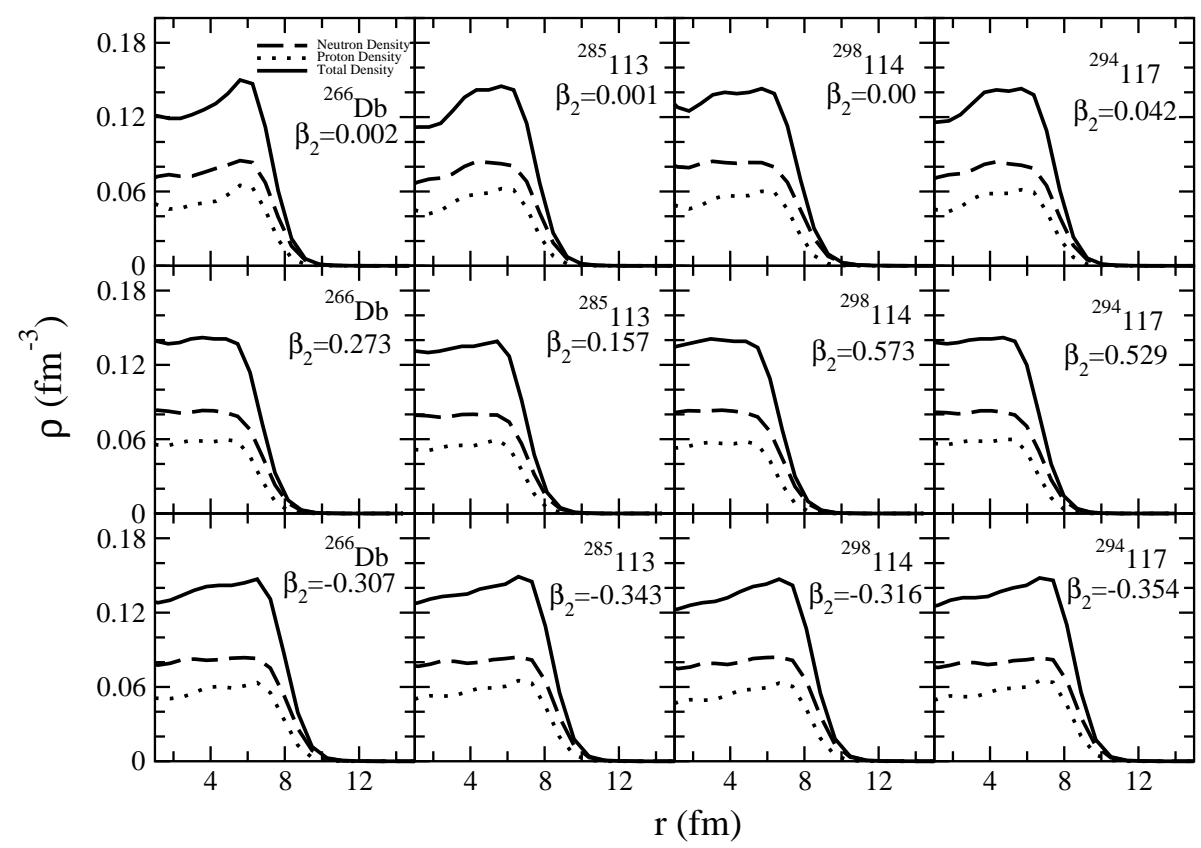

Fig. 4. Same as Fig. 3, but with RMF(NL3*).

as well as proton by using non-relativistic and relativistic densities are given in Tables I and II for all three solutions. In our present study, most of the nuclei achieve the prolate shape as their ground state solution both in the SHF and RMF calculations. Here, we do not find significant bubble structure, i.e. no good amount of depletion fraction are estimated for this shape, except a few cases. It is evident from Tables I and II, a remarkable depletion fraction is there in ${ }^{292,293} 120$ over the whole series of nuclei on spherical solution in both models.

\section{5. $Q_{\alpha}$ and $T_{\alpha}$ for superheavy nuclei}

The $Q_{\alpha}$ value of a nucleus provides valuable information for its stability. This is more important for superheavy nuclei, which is the central theme of study in the present paper. Thus, it is worthwhile to evaluate the $Q_{\alpha}$ values for the nuclei which will also give us to estimate the life time $T_{\alpha}$ of the considered nuclei. It is to be noted that mostly, $\alpha$-decay is the decay mode for these synthesized elements, which end with spontaneous fission. Recently, it is predicted that the $\beta$-decay may play an

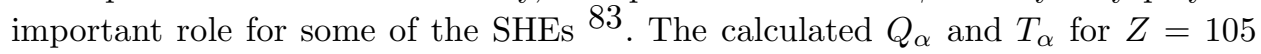
- 120 within the relativistic and non-relativistic formalism using NL3* and SkI4 parameters are listed in Table III. The $Q_{\alpha}$ is calculated by using the following 


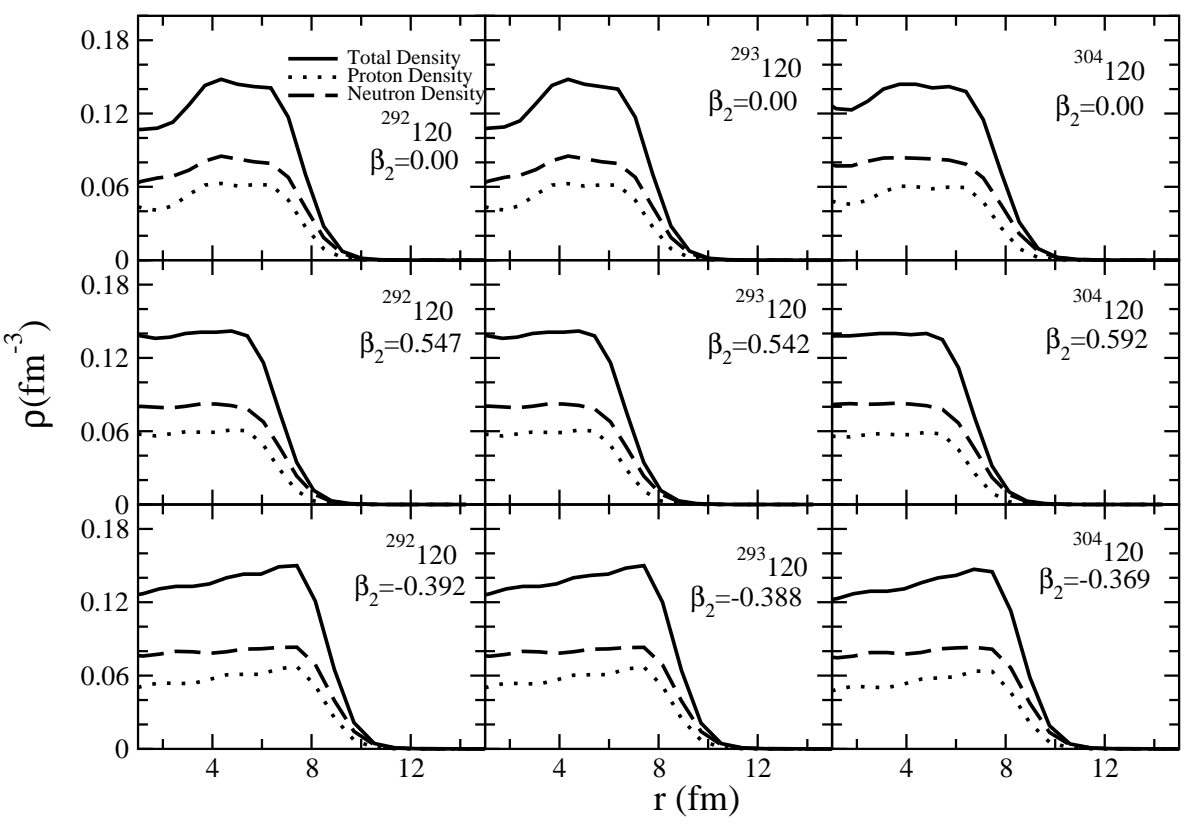

Fig. 5. Same as Fig. 3, but for ${ }^{292,293,304} 120$ with RMF(NL3*).

expression

$$
Q_{\alpha}=B E(N, Z)-B E(N-2, Z-2)-B E(2,2) .
$$

Here, $\mathrm{BE}(\mathrm{N}, \mathrm{Z})$ and $\mathrm{BE}(\mathrm{N}-2, \mathrm{Z}-2)$ are the binding energy of the parent and daughter nuclei, respectively. $B E(2,2)$ is the binding energy of $\alpha$ particle $\left({ }^{4} \mathrm{He}\right)$ i.e. 28.3 $\mathrm{MeV}$ 67. We are using the empirical Viola-Seaborg formula ${ }^{84}$ for the calculation of $T_{\alpha}$ which is define as:

$$
\log _{10}\left(T_{\alpha}\right)=\frac{(a Z+b)}{\sqrt{Q}_{\alpha}}+(c Z+d)+h \log
$$

where $\mathrm{Z}$ is the charge number of the parent nucleus and $a, b, c, d$ are the fitting constants defined as $8501.66175, b=-8.5166, c=-0.20228, d=-33.9069$. The last term in above equation is known as even-odd hindrance factor and is obtained by fits to odd nuclei. It takes the value as $h l o g=0.0$ (for $Z=$ even, $N=$ even $), h l o g=0.772($ for $Z=$ odd,$N=$ even $), h l o g=1.066($ for $Z=$ even, $N=$ $o d d), h \log =1.114($ for $Z=o d d, N=o d d)$. From the above equation, one can find that $T_{\alpha} \propto 10^{\frac{1}{\sqrt{Q}}}$, hence a small change in $Q_{\alpha}$ creates a large difference in $T_{\alpha}$. This observation reflects in our results (see Table III). 


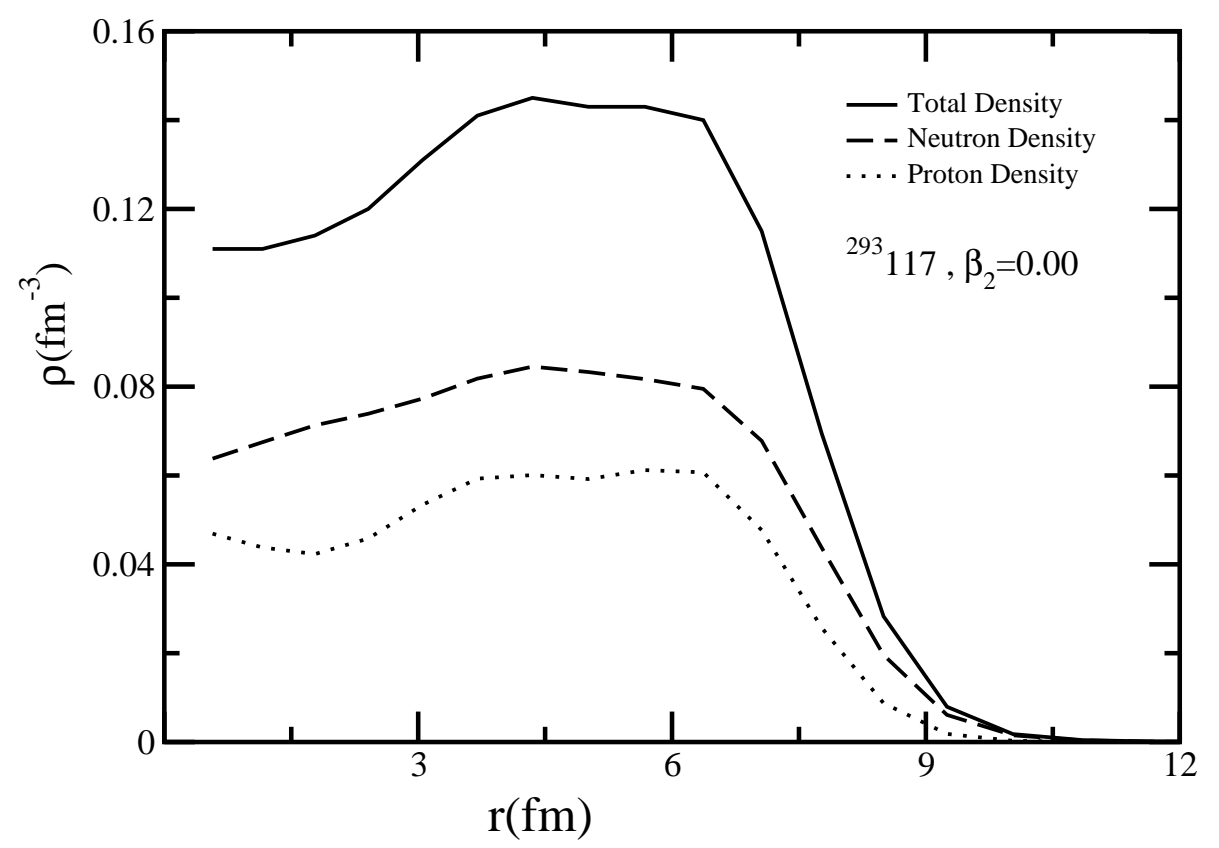

Fig. 6. The neutron, proton and total matter density distribution for ${ }^{293} 117$ with RMF(NL3*).

\section{Summary}

In summary, we study a broad spectrum of the superheavy nuclei for $Z=105$ - 120 in the frame work of relativistic and nonrelativistic mean field formalisms. The calculations are done in an axially deformed coordinates using constant gap BCS pairing. Except $Z=120$, all other isotopes are synthesized artificially whose properties are studied. From the calculated binding energies, $Q_{\alpha}$ and $T_{\alpha}$ are estimated. The results are compared with the most acceptable FRDM data as well as with the experimental observations, wherever available. Over all comparison of the results are quite agreeable with each other in all the three models along with the experimental data.

On the basis of the depletion of central density, a bubble structure is studied for some superheavy nuclei in the excited states. This observation is model independent. The maximum depletion fraction calculated in the considered nuclei is for ${ }^{292,293} 120$. In some of the cases, [Fig. [6] a depletion of proton density nearer to the center (but not at the center exactly) indicates a special type of proton distribution. In this case, the center is slightly bulgy and a considerably depression afterward. Again a big hump almost at the mid distance of the center and the surface appears in the density distribution for the spherical state. This feature of the nucleus is unique and need more investigation. Work in this direction is in progress 86 . 


\section{Acknowledgments}

One of the author (MI) acknowledge the hospitality provided by Institute of Physics, Bhubaneswar during the work.

\section{References}

1. Scharff-Goldhaber, Nucleonics 15, 122 (1957).

2. S. G. Nilsson, C. F. Tsang, A. Sobiczewski, Z. Szymanski, S. Wycech, C. Gustafson, I. -L. Lamm, P. Möller and B. Nilsson, Nucl. Phys. A 131, 1 (1969).

3. G. Herrmann, Nature 280, 543 (1979).

4. K. Kumar, Superheavy Elements (IOP Publishing Ltd. 1989); Physica Scripta, T32, 31 (1990).

5. W. D. Myers and W. J. Swiatecki, Nucl. Phys. 81, 1 (1966).

6. S. G. Nilsson, Mat. Fys. Medd. Dan. Vid. Selsk 29, 16 (1955).

7. B. R. Mottelson and S. G. Nilsson, Phys. Rep. 99, 1615 (1955); Mat. Fys. Skr. Dan. Vid. Selsk, 8, 1 (1959).

8. G. Andersson, S. E. Larsson, G. Leander, S. G. Nilsson, I. Ragnarsson and S. Aberg, Phys. Lett. B 65, 209 (1976).

9. H. W. Meldner, Ark. Fys. 36, 593 (1978).

10. H. W. Meldner, in Superheavy Elements ed. M. A. K. Lodhi (Oxford: Pergamon) p. 495.

11. H. S. Köhler, Nucl. Phys. A 162, 385 (1971).

12. V. M. Strutinsky, Nucl. Phys. A 95, 420 (1967); 122, 1 (1968).

13. P. Möller and J. R. Nix, J. Phys. G 20, 1681 (1994).

14. A. Sobiczewski, Phys. Part. Nuclei 25, 295 (1994).

15. A. Sobiczewski and K. Pomorski, Prog. Part. Nucl. Phys. 58, 292 (2007).

16. R. Smolanczuk, J. Skalski and A. Sobiczewski, Phys. Rev. C 52, 1871 (1995).

17. S. K. Patra, R. K. Gupta and W. Greiner, Mod. Phys. Lett. A 12, 1727 (1997); Tapas Sil, S.K. Patra, B.K. Sharma, M. Centelles, and X. Viñas, Phys. Rev. C 69, 044315 (2004).

18. K. Rutz, M. Bender, T. Bürvenich, T. Schilling, P.-G. Reinhardt, J. A. Maruhn, and W. Greiner, Phys. Rev. C 56, 238 (1997).

19. S. K. Patra, C.-L. Wu, C. R. Praharaj, and R. K. Gupta, Nucl. Phys. A 651, 117 (1999).

20. J. Meng, H. Toki, S. Zhou, S. Zhang, W. Long, and L. Geng, Prog. Part. Nucl. Phys. 57, 470 (2007).

21. M. Bhuyan and S. K. Patra, Mod. Phys. Lett. A 27, 1250173 (2012).

22. S. Hofmann and G. Münzenberg, Rev. Mod. Phys. 72, 733 (2000).

23. G. Münzenberg et al., Z. Phys. A 300, 107 (1981).

24. S. Hofmann et al., Z. Phys. A 350, 277 (1995).

25. S. Hofmann et al., Z. Phys. A 350, 281 (1995).

26. S. Hofmann et al., Z. Phys. A 354, 229 (1996).

27. S. Hofmann et al., Rep. Prog. Phys. 61, 639 (1998); Acta Phys. Pol. B 30, 621 (1999).

28. S. Hofmann, Russ. Chem. Rev. 78, 1123 (2009).

29. K. Morita et al., J. Phys. Soc. Jpn. 76, 043201 (2007); ibid 76, 045001 (2007)

30. Yu. Ts. Oganessian, Phys. Rev. Lett. 83, 3154 (1998).

31. Yu. Ts. Oganessian et al., Nucl. Phys. A 685, 17c (2001).

32. Yu. Ts. Oganessian et al., Phys. Rev. C 69, 021601(R) (2004).

33. Yu. Oganessian, J. Phys. G: Nucl. Part. Phys. 34, R165 (2007).

34. Yu. Ts. Oganessian et al., Phys. Rev. Lett. 104, 142502 (2010). 
35. Yu. Ts. Oganessian et al., Phys. Rev. C 83, 954315 (2011).

36. A. Türler, Eur. Phys. J. A 15, 271 (2002).

37. Ch. E. Düllmann et al., Nature 418, 859 (2002).

38. R. Eichler et al., Nature 407, 63 (2000).

39. R. Eichler et al., Nucl. Phys. A 787, 373c (2007).

40. R. Eichler et al., Radiochim. Acta 98, 133 (2010).

41. V. Pershina, Russ. Chem. Rev. 78, 1153 (2009).

42. B. Fricke and W. Greiner, Phys. Lett. B 30, 317 (1969).

43. B. Fricke, W. Greiner, J. T. Weber, Theo. Chim. Acta 21, 235 (1971).

44. V. Pershina, T. Bastug, T. Jacob, B. fricke and S. Varga, Chem. Phys. Lett. 365, 176 (2002).

45. V. Pershina and T. Bastug, Chem. Phys. 311, 139 (2005).

46. R. C. Barber, P. J. Koral, H. Nakahara, E. Vardaci and E. W. Vogt, Pure Appl. Chem. 83, 1485 (2011).

47. A. Sobiczewski and K. Pomorski, Prog. in Part. and Nucl. Phys. 58, 292 (2007).

48. J. Decharge, J.-F. Berger, K. Dietrich and M. S. Weiss, Phys. Lett. B 451, 275 (1999); J. Decharge, J.-F. Berger, M. Girod and K. Dietrich, Nucl. Phys. A 716, 55 (2003).

49. Z. Ren and H. Toki Nucl. Phys. A 689, 691 (2001).

50. Z. Ren Phys. Rev. C 65, 051304(R) (2002).

51. I. Müntian and A. Sobiczewski, Phys. Lett. B 586, 254 (2004).

52. S. Cwiok, J. Dobaczewski, P.-H. Heenen, P. Magierski and W. Nazarewicz, Nucl. Phys. A 611, 211 (1996).

53. P.-G. Reinhard and H. Flocard, Nucl. Phys. A 584, 467 (1995).

54. G. A. Lalazissis, S. Karatzikos, R. Fossion, D. Pena Arteaga,A. V. Afanasjev, and P. Ring, Phys. Lett. B, 671, 36 (2009).

55. M. Bender, P. -H. Heenen and P. -G. Reinhard, Rev. Mod. Phys. 75, 121 (2003).

56. J. R. Stone, J.C. Miller, R. Koncewicz, P.D. Stevenson, and M.R. Strayer, Phys. Rev. C 68034324 (2003).

57. M. Dutra, O. Lourenco, J. S. Sa Martins, A. Delfino, J.R. Stone and P. D. Stevenson, Phys. Rev. C 85035201 (2012).

58. E. Chabanat, P. Bonche, P. Hansel, J. Mayer, R. Schaeffer, Nucl. Phys. A 627710 (1997).

59. J. R. Stone and P.-G. Reinhard, Prog. Part. Nucl. Phys. 58, 587 (2007).

60. E. Chabanat, P. Bonche, P. Haensel, J. Meyer, and R. Schaeffer, Nucl. Phys. A 635 231 (1998).

61. B. A. Brown, Phys. Rev. C 58220 (1998).

62. B. D. Serot, Rep. Prog. Phys. 55, 1855 (1992).

63. Y. K. Gambhir, P. Ring, and A. Thimet, Ann. Phys. (N.Y.) 198, 132 (1990).

64. P. Ring, Prog. Part. Nucl. Phys. 37, 193 (1996).

65. B. D. Serot and J. D. Walecka, Adv. Nucl. Phys. 16, 1 (1986).

66. J. Boguta and A. R. Bodmer, Nucl. Phys. A 292, 413 (1977).

67. G. Audi, A.H. Wapstra and C. Thibault, Nucl. Phys. A, 729, 337 (2003).

68. P. Möller, J. R. Nix, and K.-L. Kratz, At. Data and Nucl. Data Tables 66, 131 (1997).

69. P. Möller, J. R. Nix, W. D. Myers, and W. J. Swiatecki, At. Data and Nucl. Data Tables 59, 185 (1995).

70. J. P. Maharana, Y. K. Gambhir, J. A. Sheikh and P. Ring, Phys. Rev. C 46, R1163 (1992).

71. S. K. Patra, and C. R. Praharaj, Phys. Rev. C 47, 2978 (1993).

72. F. Sarazin et al., Phys. Rev. Lett. 84, 5062 (2000).

73. J. L. Egido, L. M. Robledo, R. R. Rodriguez-Guzman, Phys. Rev. Lett. 93, 282502 
(2004).

74. J. A. Wheeler (unpublished).

75. H. A. Wilson, Phys. Rev. 69, 538 (1946).

76. P. J. Siemens and H. A. Bethe, Phys. Rev. Lett. 18, 704 (1967).

77. C. Y. Wong, Phys. Lett. B 41, 451 (1972).

78. K. T. R. Davies, C. Y. Wong and S. J. Krieger, Phys. Lett. B 41, 455 (1972).

79. J. Decharge et al., Nucl. Phys. A 716, 55 (2003).

80. M. Grasso et al., Phys. Rev. C 79, 034318 (2009).

81. W. Y. Zao. et al., Chin. Phys. Lett, 28, 102101 (2011).

82. E. Khan. et al., Nucl. Phys. A, 800, 37 (2008).

83. A. V. Karpov et al., Int. J. Mod. Phy. E 21, 1250013 (2012).

84. V. E. Viola, Jr. and G.T. Seaborg, J. Inorg. Nucl. Chem. 28, 741 (1966).

85. A. Sobiczewski, Z. Patyk and S. Cwiok, Phys. Lett. B 224, 1 (1989).

86. S. K. Singh and S. K. Patra (In progress). 